\title{
STABILIZATION OF BERGER-TIMOSHENKO'S EQUATION AS LIMIT OF THE UNIFORM STABILIZATION OF THE VON KÁRMÁN SYSTEM OF BEAMS AND PLATES
}

\author{
G. Perla Menzala ${ }^{1}$, Ademir F. Pazoto ${ }^{2}$ and Enrique Zuazua ${ }^{3}$
}

\begin{abstract}
We consider a dynamical one-dimensional nonlinear von Kármán model for beams depending on a parameter $\varepsilon>0$ and study its asymptotic behavior for $t$ large, as $\varepsilon \rightarrow 0$. Introducing appropriate damping mechanisms we show that the energy of solutions of the corresponding damped models decay exponentially uniformly with respect to the parameter $\varepsilon$. In order for this to be true the damping mechanism has to have the appropriate scale with respect to $\varepsilon$. In the limit as $\varepsilon \rightarrow 0$ we obtain damped Berger-Timoshenko beam models for which the energy tends to zero exponentially as well. This is done both in the case of internal and boundary damping. We address the same problem for plates with internal damping.
\end{abstract}

Mathematics Subject Classification. 35B40, 35Q72, 74B20.

Received: November 30, 2001. Revised: March 12, 2002.

\section{INTRODUCTION}

In this paper we investigate the decay properties of solutions for various models of damped beams and plates. Recently, it was proved that the Berger-Timoshenko beam model may be derived as a singular limit of the von Kármán beam model. This was done in $[17,18]$ in the conservative case and under various boundary conditions. Here the same analysis is developed for the corresponding damped systems.

There is a large literature on the problem of uniform rates of decay of solutions of damped beam models. This problem has been solved both in the case where the damping term is effective in the interior of the beam or through the boundary conditions. In this direction we can mention, for instance, the work by Lagnese and Leugering [11]. The works by Puel and Tucsnak $[15,16]$ and the monograph by Lagnese [8] devoted to the initial boundary value problem and the stabilization of plates are also worth mentioning.

\footnotetext{
Keywords and phrases. Uniform stabilization, singular limit, von Kármán system, beams, plates.

1 National Laboratory of Scientific Computation, LNCC/MCT, Rua Getúlio Vargas 333, Quitandinha, Petrópolis, RJ, CEP 25651-070, RJ, Brasil

and

Institute of Mathematics, UFRJ, P.O. Box 68530, Rio de Janeiro, RJ, Brasil. e-mail: perla@lncc.br

${ }^{2}$ Institute of Mathematics, PO Box 68530, Federal University of Rio de Janeiro, UFRJ, 21945-970 Rio de Janeiro, RJ, Brasil.

e-mail: ademir@acd.ufrj.br

${ }^{3}$ Departamento de Matemáticas, Universidad Autónoma de Madrid, 28049 Madrid, Spain. e-mail: enrique.zuazua@uam.es
}

(c) EDP Sciences, SMAI 2002 
It is then natural to raise the following question: Can one obtain the damped Berger-Timoshenko model as a singular limit of the damped von Kármán beam model so that the decay rates remain uniform as the singular parameter tends to zero?

To our knowledge, this problem has not been addressed in the literature and the existing developments do not allow to give an immediate answer to it. Indeed, the analysis in $[17,18]$ allows to get the convergence of solutions in bounded intervals of time. However, the decay properties we have in mind require the analysis of convergence as time goes to infinity as well.

Let us recall that Berger-Timoshenko's model describes the transversal vibrations of the beam while in von Kármán's system one also takes into account longitudinal deformations. Therefore, the problem we are considering is very closely related to the design of efficient damping mechanisms that guarantee the uniform decay of both, the longitudinal and transversal components, through the singular limiting process.

The problem we are analysing is only one example of a whole family of problems that arise in the context of vibration modelling. The connections between the various available models for a given mechanical problem may be often described precisely in mathematical terms by means of the analysis of the underlying singular perturbation problem. At this respect, in addition to the works $[17,18]$ discussed above on the beam models, we also refer to the monograph by Ciarlet [2] in which various plate models are derived as singular limits from the equations of $3-d$ elasticity in thin domains, and to [3] and [22] for the asymptotic analysis of beam models.

In all these situations the problem we address makes sense: What are the damping mechanisms that guarantee the uniform exponential decay through the singular limiting process? The problem might be easy to solve in the context of parabolic equations or, more generally, when the underlying models have a strong enough intrinsic dissipative nature. However, very often, as it is the case in the examples we address here, the models are of conservative nature and the decay requires using appropriate damping mechanisms at the right scale. Obviously, for practical purposes, it is desirable to achieve this uniform decay property with a minimal amount of damping both in what concerns its support and its intensity. Moreover, in the context of coupled systems (as it is the case in the problem we are addressing here where longitudinal and transversal vibrations are coupled), in order to achieve the desired decay property, the damping mechanism has to be designed in an appropriate way in order to capture all the components of the system. For all these reasons the right choice of damping terms is far from being obvious and requires a careful analysis in each particular case.

The dependence of the decay rate on the amount of damping is also sensitive to the possible presence of "overdamping" phenomena. Indeed, it is by now well known that, when one increases the amount of damping beyond a critical limit, the decay rate may decrease, contradicting the first intuition. Therefore, the question under consideration is even more subtle since, in a first approach, one could think that the problem would be easily solved putting a large enough amount of damping on the systems under consideration. But, due to this overdamping phenomena, this is far from reality.

In order to make more precise the problem we have in mind let us recall the essentials of $[17,18]$. In these works the following von Kármán system for the vibrations of a beam occupying the inverval $(0, L)$ was considered

$$
\begin{cases}\varepsilon v_{t t}-\left(v_{x}+\frac{1}{2} w_{x}^{2}\right)_{x}=0, & 0<x<L, t>0 \\ w_{t t}+w_{x x x x}-w_{x x t t}-\left(w_{x}\left(v_{x}+\frac{1}{2} w_{x}^{2}\right)\right)_{x}=0, & 0<x<L, t>0\end{cases}
$$

subject to various boundary conditions. In (1.1) $w=w(x, t)$ represents the transversal deformation, $v=v(x, t)$ the longitudinal one and $\varepsilon>0$ is a small parameter that is devoted to tend to zero. With appropriate boundary conditions and given initial data in suitable spaces, model (1.1) admits a unique finite energy solution $\left(v^{\varepsilon}, w^{\varepsilon}\right)$, the energy being given by

$$
E_{\varepsilon}(t)=\frac{1}{2} \int_{0}^{L}\left[\varepsilon v_{t}^{2}+\left[v_{x}+\frac{1}{2} w_{x}^{2}\right]^{2}+w_{t}^{2}+w_{x x}^{2}+w_{x t}^{2}\right] \mathrm{d} x .
$$


Moreover, this energy is constant in time. In $[17,18]$ it was proved that, as $\varepsilon \rightarrow 0$, and for appropriate boundary conditions, the solution of (1.1) is such that $w^{\varepsilon}$ converges (in an appropriate topology) towards the solution of the Berger-Timoshenko model for the transversal vibrations of the beam:

$$
w_{t t}+w_{x x x x}-w_{x x t t}-\frac{1}{2 L}\left(\int_{0}^{L} w_{x}^{2} \mathrm{~d} x\right) w_{x x}=0
$$

in which the effect of the longitudinal component has been averaged to give rise to the integral nonlinearity in (1.3).

It is however important to note that this limiting behaviour is very sensitive to the boundary conditions. Indeed, as shown in [18], in some cases, the limit $w$ obeys the linear beam equation

$$
w_{t t}+w_{x x x x}-w_{x x t t}=0
$$

There is a very natural damping term for the scalar beam models (1.3) and (1.4), namely $w_{t}-w_{x x t}$. Thus, instead of $(1.3,1.4)$, we could consider the damped models

$$
w_{t t}+w_{x x x x}-w_{x x t t}-\frac{1}{2 L}\left(\int_{0}^{L} w_{x}^{2} \mathrm{~d} x\right) w_{x x}+w_{t}-w_{x x t}=0
$$

and

$$
w_{t t}+w_{x x x x}-w_{x x t t}+w_{t}-w_{x x t}=0
$$

respectively.

It is well known that, with suitable boundary conditions, the energy of solutions of (1.5) and (1.6) decays exponentially to zero as $t \rightarrow \infty$. Therefore, the following question is in order: What is the damping mechanism in (1.1) so that when letting $\varepsilon \rightarrow 0$ one recovers (1.5) or (1.6) and such that the energy of solutions of the corresponding damped model decays exponentially uniformly (with respect to the parameter $\varepsilon \rightarrow 0$ ) to zero?

Obviously, such a damping mechanism has also to damp the longitudinal component $v^{\varepsilon}$ of the system (1.1). It is then natural to consider systems of the form

$$
\begin{cases}\varepsilon v_{t t}-\left(v_{x}+\frac{1}{2} w_{x}^{2}\right)_{x}+\varepsilon^{\alpha} v_{t}=0, & 0<x<L, t>0 \\ w_{t t}+w_{x x x x}-w_{x x t t}-\left(w_{x}\left(v_{x}+\frac{1}{2} w_{x}^{2}\right)\right)_{x}+w_{t}-w_{x x t}=0, & 0<x<L, t>0\end{cases}
$$

with $\alpha \geq 0$, in which the presence of the term $\varepsilon^{\alpha} v_{t}$ in the first equation guarantees a certain amount of damping on the longitudinal component.

In Section 3 we shall address problem (1.7) in detail. We will show that, by choosing $0<\alpha \leq 1$, the uniform exponential decay of solutions is guaranteed. To do that we shall employ a classical method consisting on introducing a suitable perturbation of the energy of the system for which one is able to obtain differential inequalities leading to the exponential decay (see, for instance, [5] and [23]). In the limit case $\alpha=0$ we shall see that the decay rate is uniform (with respect to $\varepsilon \rightarrow 0$ ) for solutions with data in balls of the energy space. Before doing this, in Section 2, we shall carefully analyze the "toy model"

$$
\varepsilon u_{t t}-u_{x x}+\varepsilon^{\alpha} u_{t}=0
$$

where explicit spectral computations may be developed. The necessity of choosing $0 \leq \alpha \leq 1$ appears then naturally. 
But, as we said above, in practice, one is interested on using damping mechanisms that are localized somewhere in the interior of the beam or on its boundary. In particular, the boundary damping case has attracted a lot of attention in the last decade. In [11] a damping mechanism for systems of the form (1.1) (with $\varepsilon=1$ ) was introduced and the exponential decay of solutions was proved. It is also by now well known that the solutions of Berger-Timoshenko's models (1.3) as well as the one for (1.4) with appropriate boundary damping mechanisms decay exponentially. Therefore, it is also natural to investigate the behavior of the decay rate as $\varepsilon \rightarrow 0$ in the presence of boundary damping terms.

In Section 4 we prove that, by an appropriate scaling of the damping terms introduced in [11], one can prove an uniform (as $\varepsilon \rightarrow 0$ ) decay rate for the solutions of (1.1). However in this case, the limiting problem (as $\varepsilon \rightarrow 0$ ) turns out to be governed by the linear parts of equation (1.3).

In Section 5 we analyze the $2-D$ case: The full nonlinear dynamic von Kármán systems with internal damping. According to the analysis in [19] it is known that the Berger-Timoshenko plate model may be obtained as a singular limit of the full von Kármán system. Here we obtain an uniform decay rate as $\varepsilon \rightarrow 0$ as in Section 3.

Finally, in Section 6 we discuss some related issues and formulate some open problems.

\section{THE LINEAR WAVE EQUATION}

Let us consider the damped wave equation with homogeneous Dirichlet boundary conditions in the inter$\operatorname{val}(0, \pi)$ :

$$
\begin{cases}\varepsilon u_{t t}-u_{x x}+\varepsilon^{\alpha} u_{t}=0, & 0<x<\pi, t>0 \\ u(0, t)=u(\pi, t)=0, & t>0 .\end{cases}
$$

Here, $\varepsilon>0$ is a small parameter, devoted to tend to zero.

Our goal is to analyze the values of $\alpha \geq 0$ for which the decay rate of the energy of solutions of (2.1), as $t \rightarrow \infty$, is uniform as $\varepsilon \rightarrow 0$.

Let us recall that the energy in (2.1) is given by

$$
E_{\varepsilon}(t)=\frac{1}{2} \int_{0}^{\pi}\left[\varepsilon u_{t}^{2}+u_{x}^{2}\right] \mathrm{d} x
$$

with the energy dissipation law being

$$
\frac{\mathrm{d} E_{\varepsilon}}{\mathrm{d} t}(t)=-\varepsilon^{\alpha} \int_{0}^{\pi} u_{t}^{2} \mathrm{~d} x
$$

Let us first compute the spectrum of (2.1). We set $u=u(x, t)=\mathrm{e}^{\lambda t} \sin (k x)$. Then, $u$ solves (2.1) if and only if $\lambda$ solves the quadratic equation

$$
\varepsilon \lambda^{2}+k^{2}+\varepsilon^{\alpha} \lambda=0
$$

i.e.,

$$
\lambda=\frac{-\varepsilon^{\alpha} \pm \sqrt{\varepsilon^{2 \alpha}-4 \varepsilon k^{2}}}{2 \varepsilon}=-\frac{\varepsilon^{\alpha-1}}{2} \pm \frac{1}{2} \sqrt{\varepsilon^{2(\alpha-1)}-4 k^{2} / \varepsilon} .
$$

When $\varepsilon^{2(\alpha-1)} \leq 4 k^{2} / \varepsilon$ the real part of the eigenvalue $\lambda$ in $(2.5)$ is $-\varepsilon^{\alpha-1} / 2$. Thus, in order to obtain a uniform (as $\varepsilon \rightarrow 0$ ) decay rate it is natural to take $\alpha$ in the range

$$
\alpha \leq 1 .
$$


When $\varepsilon^{2(\alpha-1)}-4 k^{2} / \varepsilon>0$ the eigenvalue in (2.5) with largest real part (and slowest decay) corresponds to the + sign. We then get

$$
\lambda_{+}=-\frac{\varepsilon^{\alpha-1}}{2}+\frac{1}{2} \sqrt{\varepsilon^{2(\alpha-1)}-4 k^{2} / \varepsilon}=\frac{-2 k^{2} / \varepsilon}{\varepsilon^{\alpha-1}+\sqrt{\varepsilon^{2(\alpha-1)}-4 k^{2} / \varepsilon}} \leq-\frac{k^{2}}{\varepsilon^{\alpha}} \leq-\frac{1}{\varepsilon^{\alpha}},
$$

which is in agreement with the uniform decay rate when

$$
\alpha \geq 0 .
$$

Consequently, it is natural to conjecture that the energy of solutions of (2.1) decays exponentially to zero as $t \rightarrow \infty$, uniformly as $\varepsilon \rightarrow 0$, when $0 \leq \alpha \leq 1$. To show that this is actually the case we employ the classical method of perturbing the energy $E_{\varepsilon}$ to obtain a differential inequality leading to the exponential decay $($ see $[5,23])$. This will be the main tool for analyzing the nonlinear beam models in the following sections.

We set

$$
F_{\varepsilon}(t)=\varepsilon \int_{0}^{\pi} u u_{t} \mathrm{~d} x
$$

Then

$$
\frac{\mathrm{d} F_{\varepsilon}}{\mathrm{d} t}=\int_{0}^{\pi} \varepsilon u_{t t} u \mathrm{~d} x+\varepsilon \int_{0}^{\pi} u_{t}^{2} \mathrm{~d} x=-\int_{0}^{\pi} u_{x}^{2} \mathrm{~d} x-\varepsilon^{\alpha} \int_{0}^{\pi} u u_{t} \mathrm{~d} x+\varepsilon \int_{0}^{\pi} u_{t}^{2} \mathrm{~d} x .
$$

Given $\delta>0$ we introduce the perturbed energy:

$$
G_{\varepsilon, \delta}(t)=E_{\varepsilon}(t)+\delta F_{\varepsilon}(t) .
$$

According to (2.3) and (2.9) we have

$$
\frac{\mathrm{d} G_{\varepsilon, \delta}(t)}{\mathrm{d} t}=-\delta \int_{0}^{\pi} u_{x}^{2} \mathrm{~d} x-\left(\varepsilon^{\alpha}-\delta \varepsilon\right) \int_{0}^{\pi} u_{t}^{2} \mathrm{~d} x-\delta \varepsilon^{\alpha} \int_{0}^{\pi} u u_{t} \mathrm{~d} x .
$$

Note that

$$
\left|\delta \varepsilon^{\alpha} \int_{0}^{\pi} u u_{t} \mathrm{~d} x\right| \leq \frac{\delta}{2} \int_{0}^{\pi} u^{2} \mathrm{~d} x+\frac{\delta \varepsilon^{2 \alpha}}{2} \int_{0}^{\pi} u_{t}^{2} \mathrm{~d} x \leq \frac{\delta}{2} \int_{0}^{\pi} u_{x}^{2} \mathrm{~d} x+\frac{\delta \varepsilon^{2 \alpha}}{2} \int_{0}^{\pi} u_{t}^{2} \mathrm{~d} x .
$$

The last estimate in (2.12) uses Poincaré's inequality. Combining (2.11) and (2.12) we deduce that

$$
\frac{\mathrm{d} G_{\varepsilon, \delta}(t)}{\mathrm{d} t} \leq-\frac{\delta}{2} \int_{0}^{\pi} u_{x}^{2} \mathrm{~d} x-\left(\varepsilon^{\alpha-1}-\delta-\delta \varepsilon^{2 \alpha-1} / 2\right) \varepsilon \int_{0}^{\pi} u_{t}^{2} \mathrm{~d} x .
$$

Moreover

$$
\left|F_{\varepsilon}(t)\right| \leq \sqrt{\varepsilon}\left[\frac{1}{2} \int_{0}^{\pi} u_{x}^{2} \mathrm{~d} x+\frac{\varepsilon}{2} \int_{0}^{\pi} u_{t}^{2} \mathrm{~d} x\right]=\sqrt{\varepsilon} E_{\varepsilon}(t) .
$$

Consequently

$$
\left|G_{\varepsilon, \delta}(t)-E_{\varepsilon}(t)\right| \leq \delta \sqrt{\varepsilon} E_{\varepsilon}(t) .
$$


In view of (2.13), in order to guarantee the uniform (as $\varepsilon \rightarrow 0$ ) exponential decay of the energy $E_{\varepsilon}$ it is sufficient to choose $\delta=\delta(\varepsilon)$ satisfying;

(a) $\delta \sqrt{\varepsilon} \rightarrow \ell<1$ as $\varepsilon \rightarrow 0$, so that, in view of (2.15), $G_{\varepsilon, \delta}(t)$ and $E_{\varepsilon}(t)$ are uniformly (as $\varepsilon \rightarrow 0$ ) equivalent;

(b) $\delta \geq c_{1}>0$ and $2\left(\varepsilon^{\alpha-1}-\delta-\delta \varepsilon^{2 \alpha-1} / 2\right) \geq c_{2}>0$ then, according to (2.13), one will immediately obtain

$$
\frac{\mathrm{d} G_{\varepsilon, \delta}}{\mathrm{d} t} \leq-\min \left(c_{1}, c_{2}\right) E_{\varepsilon}(t) .
$$

Let us first check (b). In order to have $2\left(\varepsilon^{\alpha-1}-\delta-\delta \varepsilon^{2 \alpha-1} / 2\right) \geq c_{2}>0$ it is sufficient to choose $\delta \leq$ $\min \left(\frac{\varepsilon^{\alpha-1}}{4}, \frac{\varepsilon^{-\alpha}}{2}\right)$ which, obviously, is compatible with the fact that $\delta \geq c_{1}>0$ for all $0 \leq \alpha \leq 1$. At this respect note that $\min \left(\frac{\varepsilon^{\alpha-1}}{4}, \frac{\varepsilon^{-\alpha}}{2}\right)$ is a constant as $\varepsilon \rightarrow 0$ when $\alpha=0,1$, while it tends to infinity when $0<\alpha<1$.

Obviously (a) is also compatible with the previous choice. Overall, it is sufficient to choose

$$
\delta \leq \min \left[\frac{\varepsilon^{\alpha-1}}{4}, \frac{\varepsilon^{\alpha}}{2}, \frac{\varepsilon^{-1 / 2}}{2}\right]
$$

to guarantee the uniform exponential decay. This is in agreement with the spectral computations made above.

Summarizing, we have seen that, by choosing $0 \leq \alpha \leq 1$, the uniform (as $\varepsilon \rightarrow 0$ ) exponential decay of the solutions of the damped wave equation (2.1) is guaranteed. We have also shown how the classical method of introducing suitable perturbations of the energy may be used to prove this result. This is of particular importance in the context of nonlinear problems in which these results may not be obtained through the analysis of the spectrum. Although our analysis has been done only for the one-dimensional case, the result also holds in any dimension. We refer to [5,7] and [23] for other developments in the context of the decay of solutions of damped wave equations.

\section{BEAM MODELS: INTERNAL DAMPING}

In this section we analyse the beam models discussed in the introduction with hinged boundary conditions and in the presence of internal damping distributed all along the beam. This is the simplest case and the analysis we need is close to the one of Section 2 on the linear wave equation but, this time, in order to obtain a damped Berger-Timoshenko's model as a singular limit when $\varepsilon \rightarrow 0$, we assume that $\alpha>0$. For $\alpha=0$ a different limit model is obtained (see Sect. 6).

Thus, for any $\varepsilon>0$ and $0<\alpha \leq 1$ we consider

$$
\begin{cases}\varepsilon v_{t t}=\left[v_{x}+\frac{1}{2} w_{x}^{2}\right]_{x}-\varepsilon^{\alpha} v_{t}, & 0<x<L, t>0 \\ w_{t t}+w_{x x x x}-w_{x x t t}=\left[\left(v_{x}+\frac{1}{2} w_{x}^{2}\right) w_{x}\right]_{x}-w_{t}+w_{x x t}, & 0<x<L, \quad t>0 \\ v(0, t)=v(L, t)=0, & t>0 \\ w(0, t)=w(L, t)=w_{x x}(0, t)=w_{x x}(L, t)=0, & t>0 \\ v(x, 0)=v_{0}(x), v_{t}(x, 0)=v_{1}(x), & 0<x<L \\ w(x, 0)=w_{0}(x), w_{t}(x, 0)=w_{1}(x), & 0<x<L .\end{cases}
$$

System (3.1) is well posed in the energy space

$$
H=H_{0}^{1}(0, L) \times L^{2}(0, L) \times\left[H^{2} \cap H_{0}^{1}(0, L)\right] \times H_{0}^{1}(0, L) .
$$


In other words, for any initial data $\left(v_{0}, v_{1}, w_{0}, w_{1}\right)$ belonging to $H$ there exists a unique finite energy solution

$$
\left(v, v_{t}, w, w_{t}\right) \in C([0, \infty) ; H)
$$

The energy of solutions of (3.1) decreases in time. Indeed, the energy is given by

$$
E_{\varepsilon}(t)=\frac{1}{2} \int_{0}^{L}\left[\varepsilon v_{t}^{2}+w_{t}^{2}+w_{x t}^{2}+w_{x x}^{2}+\left(v_{x}+\frac{1}{2} w_{x}^{2}\right)^{2}\right] \mathrm{d} x
$$

and obeys the energy dissipation law

$$
\frac{\mathrm{d} E_{\varepsilon}(t)}{\mathrm{d} t}=-\int_{0}^{L}\left[\varepsilon^{\alpha} v_{t}^{2}+w_{t}^{2}+w_{x t}^{2}\right] \mathrm{d} x .
$$

Then, for any $\left(v_{0}, v_{1}, w_{0}, w_{1}\right) \in H$ fixed, the quantities $\left(\sqrt{\varepsilon} v_{t}^{\varepsilon}, v^{\varepsilon}, w_{x x}^{\varepsilon}, w_{t}^{\varepsilon}\right)$ are uniformly bounded in $L^{\infty}\left(\mathbb{R}_{+}\right.$; $\left.L^{2}(0, L) \times H_{0}^{1}(0, L) \times L^{2}(0, L) \times H_{0}^{1}(0, L)\right)$. This uniform boundedness allows to perform the developments in $[17,18]$.

\subsection{Asymptotic limit as $\varepsilon \rightarrow \mathbf{0}$}

For the sake of completeness, we fix $\alpha>0$ and briefly recall the main steps of the proof of the convergence result in $[17,18]$ for the model under consideration. Here, $\left\{v^{\varepsilon}, w^{\varepsilon}\right\}$ denotes the solution-pair of system (3.1). Thus, it turns out that, according to (3.5), the following sequences (in $\varepsilon$ ) remain bounded in $L^{\infty}\left(0,+\infty ; L^{2}(0, L)\right)$ :

$$
\left\{\sqrt{\varepsilon} v_{t}^{\varepsilon}\right\},\left\{v_{x}^{\varepsilon}+\frac{1}{2}\left(w_{x}^{\varepsilon}\right)^{2}\right\},\left\{w_{t}^{\varepsilon}\right\},\left\{w_{x t}^{\varepsilon}\right\},\left\{w_{x x}^{\varepsilon}\right\} .
$$

Extracting subsequences (that we still denote by the index $\varepsilon$ in order to simplify notations), we deduce that there exist $\xi(x, t), \eta(x, t)$ and $w(x, t)$ such that

$$
\begin{gathered}
\sqrt{\varepsilon} v_{t}^{\varepsilon} \rightarrow \xi \quad \text { weakly * in } \quad L^{\infty}\left(0,+\infty ; L^{2}(0, L)\right) \\
v_{x}^{\varepsilon}+\frac{1}{2}\left(w_{x}^{\varepsilon}\right)^{2} \rightarrow \eta \quad \text { weakly * in } \quad L^{\infty}\left(0,+\infty ; L^{2}(0, L)\right) \\
w^{\varepsilon} \rightarrow w \quad \text { weakly * in } L^{\infty}\left(0,+\infty ; H^{2}(0, L)\right) \cap W^{1, \infty}\left(0,+\infty ; H_{0}^{1}(0, L)\right)
\end{gathered}
$$

as $\varepsilon \rightarrow 0$.

Clearly, the weak convergence in (3.8) suffices to pass the limit in the linear terms of (3.1). It remains to identify the weak limit of the nonlinear term

$$
\left[w_{x}^{\varepsilon}\left(v_{x}^{\varepsilon}+\frac{1}{2}\left(w_{x}^{\varepsilon}\right)^{2}\right)\right]_{x}
$$

as $\varepsilon \rightarrow 0$. Since $E_{\varepsilon}(t)$ is bounded, then $\left\{w^{\varepsilon}\right\}_{\varepsilon>0}$ is uniformly bounded in

$$
L^{\infty}\left(0,+\infty ; H^{2} \cap H_{0}^{1}(0, L)\right) \cap W^{1, \infty}\left(0, \infty ; H_{0}^{1}(0, L)\right) .
$$

Then we can use the Aubin-Lions compactness criteria (see e.g. [21]) to deduce that

$$
w^{\varepsilon} \rightarrow w \quad \text { strongly in } \quad L^{\infty}\left(0, T ; H^{2-\delta}(0, L)\right)
$$


as $\varepsilon \rightarrow 0$, for any $\delta>0$ and $T<\infty$. Combining (3.7) and (3.9), it follows that

$$
w_{x}^{\varepsilon}\left[v_{x}^{\varepsilon}+\frac{1}{2}\left(w_{x}^{\varepsilon}\right)^{2}\right] \rightarrow w_{x} \eta \quad \text { weakly in } \quad L^{2}((0, L) \times(0, T))
$$

as $\varepsilon \rightarrow 0$ for any $T<\infty$. The next step is to identify the weak limit $\eta$ in (3.7). Again, we use the boundedness of $E_{\varepsilon}(t)$ to observe that $\left\{v_{x}^{\varepsilon}\right\}$ is bounded in $L^{2}((0, L) \times(0, T))$. Consequently, we can extract a subsequence such that

$$
v_{x}^{\varepsilon} \rightarrow \rho \quad \text { weakly in } \quad L^{2}((0, L) \times(0, T))
$$

as $\varepsilon \rightarrow 0$ for some $\rho=\rho(x, t)$. From (3.9) and (3.11), we deduce that

$$
v_{x}^{\varepsilon}+\frac{1}{2}\left(w_{x}^{\varepsilon}\right)^{2} \rightarrow \rho+\frac{1}{2} w_{x}^{2} \quad \text { weakly in } \quad L^{2}((0, L) \times(0, T))
$$

which, together with (3.7), implies that

$$
\eta=\rho+\frac{1}{2} w_{x}^{2}
$$

We claim that $\eta$ is independent of $x$. In fact, since $\alpha>0$, due to (3.5) and Poincaré's inequality we can bound $\left\{v^{\varepsilon}\right\}$ in $L^{\infty}\left(0, T ; H_{0}^{1}(0, L)\right)$ to obtain that

$$
\varepsilon^{\alpha} v_{t} \rightarrow 0 \quad \text { weakly in } \quad H^{-1}\left(0, T ; H_{0}^{1}(0, L)\right)
$$

as $\varepsilon \rightarrow 0$. On the other hand, due to (3.6),

$$
\varepsilon v_{t t} \rightarrow 0 \quad \text { weakly in } \quad H^{-1}\left(0, T ; L^{2}(0, L)\right)
$$

as $\varepsilon \rightarrow 0$. From the first equation in $(3.1,3.14,3.15)$ and $(3.12)$ it follows that

$$
\eta_{x}=\left[\rho+\frac{1}{2} w_{x}^{2}\right]_{x}=0
$$

which proves our claim. Thus $\eta=\eta(t)$. Integrating identity (3.13) from $x=0$ to $x=L$, we get

$$
\eta(t) L=\int_{0}^{L} \rho \mathrm{d} x+\frac{1}{2} \int_{0}^{L} w_{x}^{2} \mathrm{~d} x=\frac{1}{2} \int_{0}^{L} w_{x}^{2} \mathrm{~d} x
$$

because $\int_{0}^{L} \rho \mathrm{d} x=0$. Indeed,

$$
\int_{0}^{L} \rho \mathrm{d} x=\lim _{\varepsilon \rightarrow 0} \int_{0}^{L} v_{x}^{\varepsilon} \mathrm{d} x=0,
$$

since $v^{\varepsilon}(0, t)=v^{\varepsilon}(L, t)=0$ and (3.11) holds. Hence

$$
\eta w_{x}=\left(\frac{1}{2 L} \int_{0}^{L} w_{x}^{2} \mathrm{~d} x\right) w_{x} .
$$


Consequently,

$$
\left[w_{x}^{\varepsilon}\left(v_{x}^{\varepsilon}+\frac{1}{2}\left(w_{x}^{\varepsilon}\right)^{2}\right)\right]_{x} \rightarrow\left(\frac{1}{2 L} \int_{0}^{L} w_{x}^{2} \mathrm{~d} x\right) w_{x x} \quad \text { weakly in } \quad L^{2}\left(0, T ; H^{-1}(0, L)\right)
$$

as $\varepsilon \rightarrow 0$.

To conclude our result, it suffices to identify the initial data of the limit system: we know that $w^{\varepsilon}(x, 0)=$ $w_{0}(x), \quad 0<x<L$ and $0<\varepsilon<1$. On the other hand, since $w^{\varepsilon} \rightarrow w$ in $C\left([0, T] ; H^{2-\delta}(0, L)\right)$ as $\varepsilon \rightarrow 0$, for any $\delta>0$ and $T<\infty, w^{\varepsilon}(x, 0) \rightarrow w(x, 0), 0<x<L$. Combining these facts we get $w(x, 0)=w_{0}(x), 0<x<L$. In order to prove that $w_{t}(x, 0)=w_{1}(x)$ we proceed in a similar way, using that $w_{t}^{\varepsilon}(x, 0)=w_{1}(x), 0<x<L$ and $0<\varepsilon<1$, and that $w_{t}^{\varepsilon} \rightarrow w_{t}$ in $C\left([0, T] ; L^{2}(0, L)\right)$. To obtain this last convergence we use the Aubin-Lions criteria (see [21]) since

$$
\left\{w_{t}^{\varepsilon}\right\} \quad \text { is bounded in } \quad L^{\infty}\left(0, T ; H_{0}^{1}(0, L)\right)
$$

and

$$
\left\{w_{t t}^{\varepsilon}\right\} \quad \text { is bounded in } \quad L^{\infty}\left(0, T ; L^{2}(0, L)\right)
$$

for $0<T<\infty$. The last bound can be easily obtained using identity

$$
w_{t t}=-\left(I-\frac{\mathrm{d}^{2}}{\mathrm{~d} x^{2}}\right)^{-1}\left(w_{x x x x}-w_{t}-w_{x x t}-\left[\left(v_{x}+\frac{1}{2} w_{x}^{2}\right) w_{x}\right]_{x}\right)
$$

together with the boundary conditions satisfied by $w^{\varepsilon}$ and taking into acount that $w^{\varepsilon}, w_{t}^{\varepsilon}$ and $\left(v_{x}^{\varepsilon}+\frac{1}{2}\left(w_{x}^{\varepsilon}\right)^{2}\right) w_{x}^{\varepsilon}$ are bounded in $L^{\infty}\left(0, T ; H^{2}(0, L)\right), \quad L^{\infty}\left(0, T ; H^{1}(0, L)\right)$ and $L^{\infty}\left(0, T ; H^{1}(0, L)\right)$ respectively.

The above convergences hold along suitable subsequences. However, taking into account that the limit $w$ has been identified as the unique solution of

$$
\begin{aligned}
& w_{t t}-w_{x x t t}+w_{x x x x}-\left(\frac{1}{2 L} \int_{0}^{L} w_{x}^{2} \mathrm{~d} x\right) w_{x x}+w_{t}-w_{x x t}=0 \text { in }(0, L) \times(0,+\infty) \\
& w(0, t)=w(L, t)=w_{x x}(0, t)=w_{x x}(L, t)=0, \quad t>0 \\
& w(x, 0)=w_{0}(x), w_{t}(x, 0)=w_{1}(x), x \in(0, L)
\end{aligned}
$$

we deduce that the whole family converges as $\varepsilon \rightarrow 0$. The energy of the limit system (3.16) is given by

$$
E(t)=\frac{1}{2} \int_{0}^{L}\left[w_{t}^{2}+w_{x x}^{2}+w_{x t}^{2}\right] \mathrm{d} x+\frac{1}{8 L}\left[\int_{0}^{L} w_{x}^{2} \mathrm{~d} x\right]^{2}
$$

and it is dissipated according to the law

$$
\frac{\mathrm{d} E}{\mathrm{~d} t}(t)=-\int_{0}^{L}\left[w_{t}^{2}+w_{x t}^{2}\right] \mathrm{d} x
$$

It is easy to see that both, for system (3.1) and (3.16), the energy decays exponentially as $t \rightarrow+\infty$. Our goal here is to show that, actually, the decay rate is uniform (as $\varepsilon \rightarrow 0$ ), provided $0<\alpha \leq 1$ and locally uniformly for bounded data when $\alpha=0$. Although, as we said above, when $\alpha=0$ the limit system as $\varepsilon \rightarrow 0$ is different. This will be analized in Section 6 . 


\subsection{Uniform stabilization as $\varepsilon \rightarrow \mathbf{0}$}

The following result holds:

Theorem 3.1. Let $\{v, w\}$ be the global solution of problem (3.1) with initial data in the space H. Assume that $0 \leq \alpha \leq 1$. Then, there exist positive constants $c>0, \mu>0$, such that

$$
E_{\varepsilon}(t) \leq c E_{\varepsilon}(0) \mathrm{e}^{-\frac{\mu}{1+\varepsilon^{\alpha} E_{\varepsilon}(0)} t}, \quad \forall t>0
$$

for all $0<\varepsilon<1$.

Remark. As a limit of (3.19) when $\varepsilon \rightarrow 0$, we obtain the following decay rates for the limit system (3.16): There exist positive constants $c, \mu>0$ such that

$$
\begin{aligned}
& \text { - If } \alpha=0: E(t) \leq c \mathrm{e}^{-\frac{\mu}{1+E(0)} t} E(0), \forall t>0, \\
& \text { - If } \alpha>0: E(t) \leq c \mathrm{e}^{-\mu t} E(0), \forall t>0,
\end{aligned}
$$

for every solution with initial data in the energy space. Obviously (3.21) is better than (3.20) since it holds uniformly on all solutions while (3.20) is only uniform on bounded sets of the energy space. However, as we shall see in Section 6 , when $\alpha=0$, the limit equation is not (3.16). Note also that, for $\varepsilon>0$, the decay estimate (3.19) is only uniform in bounded sets of initial data. However, when $\alpha>0$, as $\varepsilon \rightarrow 0$, the dependence on the data is weaker and, in the limit, we get the uniform decay property (3.21).

Proof of Theorem 3.1. In order to simplify notations we write $w=w^{\varepsilon}$ and $v=v^{\varepsilon}$. We will prove the theorem in three steps:

Step 1. Let us consider the functional

$$
F_{\varepsilon}(t)=\varepsilon \int_{0}^{L} v v_{t} \mathrm{~d} x+\frac{1}{2} \int_{0}^{L}\left[w w_{t}+w_{x} w_{x t}\right] \mathrm{d} x .
$$

Using the equations in (3.1) it follows that

$$
\begin{aligned}
\frac{\mathrm{d} F_{\varepsilon}(t)}{\mathrm{d} t}= & \varepsilon \int_{0}^{L} v_{t}^{2} \mathrm{~d} x+\varepsilon \int_{0}^{L} v v_{t t} \mathrm{~d} x+\frac{1}{2} \int_{0}^{L}\left[w_{t}^{2}+w_{x t}^{2}\right] \mathrm{d} x+\frac{1}{2} \int_{0}^{L}\left[w w_{t t}+w_{x} w_{x t t}\right] \mathrm{d} x \\
= & \varepsilon \int_{0}^{L} v_{t}^{2} \mathrm{~d} x+\int_{0}^{L} v\left[v_{x}+\frac{1}{2} w_{x}^{2}\right]_{x} \mathrm{~d} x-\varepsilon^{\alpha} \int_{0}^{L} v v_{t} \mathrm{~d} x+\frac{1}{2} \int_{0}^{L}\left[w_{t}^{2}+w_{x t}^{2}\right] \mathrm{d} x \\
& +\frac{1}{2} \int_{0}^{L} w\left[-w_{x x x x}+\left[\left(v_{x}+\frac{1}{2} w_{x}^{2}\right) w_{x}\right]_{x}-w_{t}+w_{x x t}\right] \mathrm{d} x \\
= & \varepsilon \int_{0}^{L} v_{t}^{2} \mathrm{~d} x-\int_{0}^{L}\left[v_{x}+\frac{1}{2} w_{x}^{2}\right]^{2} \mathrm{~d} x+\frac{1}{2} \int_{0}^{L}\left[w_{t}^{2}+w_{x t}^{2}\right] \mathrm{d} x \\
& -\frac{1}{2} \int_{0}^{L} w_{x x}^{2} \mathrm{~d} x+\frac{1}{2} \int_{0}^{L} w\left[w_{x x t}-w_{t}\right] \mathrm{d} x-\varepsilon^{\alpha} \int_{0}^{L} v v_{t} \mathrm{~d} x .
\end{aligned}
$$

Moreover

$$
\left|\int_{0}^{L} w w_{t} \mathrm{~d} x\right| \leq c \int_{0}^{L}\left[\eta w_{x x}^{2}+\frac{1}{\eta} w_{t}^{2}\right] \mathrm{d} x
$$


for all $\eta>0$, since $\left\|w_{x x}\right\|_{L^{2}(0, L)}$ defines a norm in $H^{2} \cap H_{0}^{1}(0, L)$ which is equivalent to the one induced by $H^{2}(0, L)$. On the other hand,

$$
\left|\int_{0}^{L} w w_{x x t} \mathrm{~d} x\right|=\left|\int_{0}^{L} w_{x} w_{x t} \mathrm{~d} x\right| \leq c \int_{0}^{L}\left[\eta w_{x x}^{2}+\frac{1}{\eta} w_{x t}^{2}\right] \mathrm{d} x
$$

and

$$
\varepsilon^{\alpha}\left|\int_{0}^{L} v v_{t} \mathrm{~d} x\right| \leq \frac{\varepsilon^{\alpha}}{2 \eta} \int_{0}^{L} v_{t}^{2} \mathrm{~d} x+\frac{\varepsilon^{\alpha} \eta}{2} \int_{0}^{L} v^{2} \mathrm{~d} x
$$

Moreover,

$$
\begin{aligned}
\int_{0}^{L} v^{2} \mathrm{~d} x & \leq c \int_{0}^{L} v_{x}^{2} \mathrm{~d} x \leq 2 c \int_{0}^{L}\left[\left[v_{x}+\frac{1}{2} w_{x}^{2}\right]^{2}+w_{x}^{4}\right] \mathrm{d} x \\
& \leq c_{1}\left[\int_{0}^{L}\left[v_{x}+\frac{1}{2} w_{x}^{2}\right]^{2} \mathrm{~d} x+\left(\int_{0}^{L} w_{x x}^{2} \mathrm{~d} x\right)^{2}\right] \\
& \leq c_{1}\left[\int_{0}^{L}\left[v_{x}+\frac{1}{2} w_{x}^{2}\right]^{2} \mathrm{~d} x+E_{\varepsilon}(0) \int_{0}^{L} w_{x x}^{2} \mathrm{~d} x\right]
\end{aligned}
$$

for some positive constant $c_{1}$.

Step 2. Now, we set

$$
G_{\varepsilon, \delta}(t)=E_{\varepsilon}(t)+\delta F_{\varepsilon}(t)
$$

with $\delta>0$. Combining (3.5) and (3.23) up to (3.27) we obtain

$$
\begin{aligned}
\frac{\mathrm{d} G_{\varepsilon, \delta}(t)}{\mathrm{d} t} \leq & -\left(\varepsilon^{\alpha-1}-\delta-\frac{\delta \varepsilon^{\alpha-1}}{2 \eta}\right) \varepsilon \int_{0}^{L} v_{t}^{2} \mathrm{~d} x-\left(1-\frac{c_{1} \delta}{\eta}\right) \int_{0}^{L}\left[w_{t}^{2}+w_{x t}^{2}\right] \mathrm{d} x \\
& -\delta\left(1-c_{1} \varepsilon^{\alpha} \eta\right) \int_{0}^{L}\left[v_{x}+\frac{1}{2} w_{x}^{2}\right]^{2} \mathrm{~d} x-\frac{\delta}{2}\left(1-c_{1} \eta\left(1+\varepsilon^{\alpha} E_{\varepsilon}(0)\right)\right) \int_{0}^{L} w_{x x}^{2} \mathrm{~d} x .
\end{aligned}
$$

We now choose $\eta$ of the order of

$$
\eta=\frac{\lambda}{1+\varepsilon^{\alpha} E_{\varepsilon}(0)}
$$

with $\lambda>0$ (small enough but independent of $\varepsilon$ and $E_{\varepsilon}(0)$ ) so that (3.29) reads as follows:

$$
\begin{aligned}
\frac{\mathrm{d} G_{\varepsilon, \delta}}{\mathrm{d} t}(t) \leq & -\left(\varepsilon^{\alpha-1}-\delta-\frac{\delta}{2 \lambda}\left(1+\varepsilon^{\alpha} E_{\varepsilon}(0)\right) \varepsilon^{\alpha-1}\right) \varepsilon \int_{0}^{L} v_{t}^{2} \mathrm{~d} x \\
& -\left(1-\frac{c_{1} \delta}{\lambda}\left(1+\varepsilon^{\alpha} E_{\varepsilon}(0)\right)\right) \int_{0}^{L}\left[w_{t}^{2}+w_{x t}^{2}\right] \mathrm{d} x \\
& -\frac{\delta}{2} \int_{0}^{L}\left[v_{x}+\frac{1}{2} w_{x}^{2}\right]^{2} \mathrm{~d} x-\frac{\delta}{2}\left(1-c_{1} \lambda\right) \int_{0}^{L} w_{x x}^{2} \mathrm{~d} x
\end{aligned}
$$

(because $\left.\eta \leq\left(c_{1} \varepsilon^{\alpha}\right)^{-1}\right)$. 
Let us impose the following restrictions on $\delta$ :

$$
\frac{c_{1}}{\lambda} \delta\left(1+\varepsilon^{\alpha} E_{\varepsilon}(0)\right) \leq \frac{1}{2}
$$

and

$$
\varepsilon^{\alpha-1}-\delta-\frac{1}{2 \lambda} \delta\left(1+\varepsilon^{\alpha} E_{\varepsilon}(0)\right) \varepsilon^{\alpha-1} \geq \frac{1}{2} .
$$

If these conditions hold, in view of (3.31), we deduce that

$$
\frac{\mathrm{d} G_{\varepsilon, \delta}(t)}{\mathrm{d} t} \leq-\min (1, \delta) E_{\varepsilon}(t)
$$

Conditions $(3.32,3.33)$ hold when

$$
\delta \leq c \min \left[\frac{1}{1+\varepsilon^{\alpha} E_{\varepsilon}(0)}, \varepsilon^{\alpha-1}\right]
$$

with $c>0$ small enough, independent of $0<\varepsilon<1$ and the solution. Observe that (3.34) holds automatically if

$$
\delta \leq \frac{c}{1+\varepsilon^{\alpha} E_{\varepsilon}(0)}
$$

with $c>0$ small but independent of $0<\varepsilon<1$ and the solution.

Step 3. To get the exponential decay of $E_{\varepsilon}$ using (3.34) we need to compare $E_{\varepsilon}$ and $G_{\varepsilon, \delta}$. To do that we use $(3.24,3.25)$ and $(3.27)$ to obtain

$$
\begin{aligned}
\left|F_{\varepsilon}(t)\right| & \leq \frac{\varepsilon}{2} \int_{0}^{L} v_{t}^{2} \mathrm{~d} x+\frac{\varepsilon}{2} \int_{0}^{L} v^{2} \mathrm{~d} x+c \int_{0}^{L}\left(w_{t}^{2}+w_{x t}^{2}+w_{x x}^{2}\right) \mathrm{d} x \\
& \leq c\left[E_{\varepsilon}(t)+\varepsilon E_{\varepsilon}^{2}(t)\right] \leq c\left[1+\varepsilon E_{\varepsilon}(0)\right] E_{\varepsilon}(t)
\end{aligned}
$$

with $c>0$ independent of $0<\varepsilon<1$ and the solution. Consequently

$$
\left|G_{\varepsilon, \delta}(t)-E_{\varepsilon}(t)\right|=\delta\left|F_{\varepsilon}(t)\right| \leq c \delta\left(1+\varepsilon E_{\varepsilon}(0)\right) E_{\varepsilon}(t) .
$$

Then, with the choice of $\delta$ as above and taking, if needed, $c>0$ smaller, we can guarantee that

$$
\left|G_{\varepsilon, \delta}(t)-E_{\varepsilon}(t)\right| \leq \frac{1}{2} E_{\varepsilon}(t)
$$

and therefore, in view of (3.34),

$$
\frac{\mathrm{d} G_{\varepsilon, \delta}}{\mathrm{d} t}(t) \leq-\frac{1}{2} \min (1, \delta) G_{\varepsilon, \delta}(t) .
$$

As a consequence of (3.40), (3.39) and the choice of $\delta$ in (3.36) we deduce the existence of positive constants $\tilde{c}>0$ and $\mu>0$ such that

$$
E_{\varepsilon}(t) \leq \tilde{c} E_{\varepsilon}(0) \mathrm{e}^{-\frac{\mu t}{1+\varepsilon^{\alpha} E(0)}}, \quad \forall t>0
$$

for every solution of (3.1) and every $0<\varepsilon<1$. 


\subsection{Other boundary conditions}

Theorem 3.1 remains valid when we consider model (3.1) with Neumann conditions on $v$ and clamped ends for $w$ :

$$
\begin{cases}v_{x}(0, t)=v_{x}(L, t)=0, & \forall t>0 \\ w(0, t)=w(L, t)=w_{x}(0, t)=w_{x}(L, t)=0, & \forall t>0 .\end{cases}
$$

Thanks to (3.5) we obtain uniform bounds for the solution which allow us to pass to the limit as $\varepsilon \rightarrow 0$ as in the previous section. Again, the only difficulty to determine the weak limit is the identification of the limit of the nonlinear term $w_{x}^{\varepsilon}\left(v_{x}^{\varepsilon}+\frac{1}{2}\left(w_{x}^{\varepsilon}\right)^{2}\right)$. Proceeding as in the previous section it follows that

$$
w_{x}^{\varepsilon}\left(v_{x}^{\varepsilon}+\frac{1}{2}\left(w_{x}^{\varepsilon}\right)^{2}\right) \rightarrow w_{x}\left(\rho+\frac{1}{2} w_{x}^{2}\right)
$$

weakly in $L^{2}((0, L) \times(0, T))$ as $\varepsilon \rightarrow 0$, and $\eta=\rho+\frac{1}{2} w_{x}^{2}$ is independent of $x$. It remains to identify the value of $\eta(t)$. We take the derivative in $x$ in the first equation of $(3.1)$ and multiply the result by $a(x)=$ $\frac{1}{4} L^{2}-\left(x-\frac{1}{2} L\right)^{2}$. Integration (in space) from zero to $L$ followed by integration by parts yields

$$
\begin{aligned}
\varepsilon \frac{\mathrm{d}^{2}}{\mathrm{~d} t^{2}} \int_{0}^{L} v_{x}^{2} a(x) \mathrm{d} x & =\int_{0}^{L}\left[v_{x}^{\varepsilon}+\frac{1}{2}\left(w_{x}^{\varepsilon}\right)^{2}\right]_{x x} a(x) \mathrm{d} x-\varepsilon^{\alpha} \frac{\mathrm{d}}{\mathrm{d} t} \int_{0}^{L} v_{x}^{2} a(x) \mathrm{d} x \\
& =-2 \int_{0}^{L}\left[v_{x}^{\varepsilon}+\frac{1}{2}\left(w_{x}^{\varepsilon}\right)^{2}\right] \mathrm{d} x-\varepsilon^{\alpha} \frac{\mathrm{d}}{\mathrm{d} t} \int_{0}^{L} v_{x}^{2} a(x) \mathrm{d} x .
\end{aligned}
$$

Note that, when integrating by parts, no boundary terms appear since $a=0$ at $x=0, L$ and also because of the boundary conditions that $v^{\varepsilon}$ and $w^{\varepsilon}$ satisfy which guarantee us that $v_{x}^{\varepsilon}+\frac{1}{2}\left(w_{x}^{\varepsilon}\right)^{2}=0$ at $x=0, L$. Since $a \in L^{2}(0, L)$, passing to the limit as $\varepsilon \rightarrow 0$, we have

$$
\int_{0}^{L} v_{x}^{\varepsilon} a(x) \mathrm{d} x \rightarrow \int_{0}^{L} \rho a(x) \mathrm{d} x \quad \text { weakly in } \quad L^{2}(0, T) .
$$

On the other hand,

$$
\varepsilon \frac{\mathrm{d}^{2}}{\mathrm{~d} t^{2}} \int_{0}^{L} v_{x}^{\varepsilon} a(x) \mathrm{d} x \rightarrow 0 \quad \text { and } \quad \frac{\mathrm{d}}{\mathrm{d} t} \varepsilon^{\alpha} \int_{0}^{L} v_{x}^{\varepsilon} \mathrm{d} x \rightarrow 0
$$

in $D^{\prime}(0, T)$ as $\varepsilon \rightarrow 0$. Finally, passing to the limit in identity (3.42) we deduce that

$$
-2 L \eta(t)=0
$$

Thus $\eta \equiv 0$. Consequently all the analysis of this section may be carried out under the boundary condition (3.41). The uniform exponential decay of the energy holds when $0<\alpha \leq 1$. However, there is a difference with respect to the previous case since the limit equation is now linear:

$$
\begin{cases}w_{t t}+w_{x x x x}-w_{x x t t}+w_{t}-w_{x x t}=0, & 0<x<L, t>0 \\ w(0, t)=w(L, t)=w_{x}(0, t)=w_{x}(L, t)=0, & t>0, \\ w(x, 0)=w_{0}(x), w_{t}(x, 0)=w_{1}(x), & 0<x<L .\end{cases}
$$




\section{BOUNDARY DAMPING ON THE TRANSVERSAL AND LONGITUDINAL COMPONENTS}

In this section we will analyze the beam model in the case where the energy of the transversal deformation as well as the longitudinal component $v$ are dissipated by means of boundary damping mechanisms. We use the same damping terms as in [11]:

$$
\begin{cases}\varepsilon v_{t t}=\left[v_{x}+\frac{1}{2} w_{x}^{2}\right]_{x}, & 0<x<L, t>0 \\ w_{t t}+w_{x x x x}-w_{x x t t}=\left[\left(v_{x}+\frac{1}{2} w_{x}^{2}\right) w_{x}\right]_{x}, & 0<x<L, t>0 \\ v(0, t)=w(0, t)=w_{x}(0, t)=0, & t>0 \\ {\left[v_{x}+\frac{1}{2} w_{x}^{2}\right](L, t)=-\varepsilon^{\alpha} v_{t}(L, t),} & t>0 \\ w_{x x}(L, t)=-w_{x t}(L, t), & t>0 \\ {\left[w_{x x x}-w_{x t t}-\left(v_{x}+\frac{1}{2} w_{x}^{2}\right) w_{x}\right](L, t)=w_{t}(L, t),} & t>0 \\ v(x, 0)=v_{0}(x), v_{t}(x, 0)=v_{1}(x), & 0<x<L \\ w(x, 0)=w_{0}(x), w_{t}(x, 0)=w_{1}(x), & 0<x<L .\end{cases}
$$

The energy of this system is dissipated and, as we shall see, the analysis of the uniform (with respect to $\varepsilon$ with $0<\varepsilon<1$ ) exponential decay of the energy may be performed as in the previous sections. On the other hand, proceeding as in $[17,18]$ and Section 3.2 we show that the limit of solutions of $(4.1)$ as $\varepsilon \rightarrow 0$ is the solution of the linear Berger-Timoshenko beam model with boundary dissipation when $\alpha>0$. The limit model when $\alpha=0$ is again of different nature and will be discussed in Section 6 .

Let us now briefly recall the well-posedness of system (4.1).

\subsection{Existence and uniqueness of solutions}

We introduce the Hilbert space

$$
H=V \times L^{2}(0, L) \times W \times V
$$

where

$$
V=\left\{\varphi \in H^{1}(0, L): \varphi(0)=0\right\}, \quad W=\left\{\varphi \in H^{2}(0, L): \varphi(0)=\varphi_{x}(0)=0\right\} .
$$

The norm in $H$ is given by

$$
\|(v, y, w, z)\|_{H}^{2}=\|v\|_{H^{1}(0, L)}^{2}+\varepsilon\|y\|^{2}+\left\|w_{x x}\right\|^{2}+\|z\|_{H^{1}(0, L)}^{2}
$$

for any $(v, y, w, z) \in H$. Here, $\|\cdot\|$ denotes the norm in $L^{2}(0, L)$.

In the sequel, we present some basic results obtained in [11] concerning existence and uniqueness of solutions for system (4.1). Concerning strong solutions, the following holds:

Theorem 4.1 (Strong solutions). Let $\left(v_{0}, v_{1}, w_{0}, w_{1}\right) \in\left[H^{2}(0, L) \cap V\right] \times V \times\left[H^{3}(0, L) \cap W\right] \times W$ with $v_{0, x}(L)+$ $\frac{1}{2}\left(w_{0, x}(L)\right)^{2}=-\varepsilon^{\alpha} v_{1}(L)$ and $w_{0, x x}(L)=w_{1, x}(L)$. Then, there exists only one pair $\{v, w\}$ satisfying

$$
\begin{aligned}
& v \in L_{\mathrm{loc}}^{\infty}\left(0,+\infty ; H^{2}(0, L) \cap V\right), v_{t} \in L_{\mathrm{loc}}^{\infty}(0,+\infty ; V) \\
& w \in L_{\mathrm{loc}}^{\infty}\left(0,+\infty ; H^{3}(0, L) \cap W\right), w_{t} \in L_{\mathrm{loc}}^{\infty}(0,+\infty ; W)
\end{aligned}
$$


and

$$
\begin{gathered}
\varepsilon v_{t t}=\left[v_{x}+\frac{1}{2} w_{x}^{2}\right]_{x}, \text { a.e. in }(x, t) \in(0, L) \times(0, \infty) \\
\left(w_{t t}, \phi\right)_{H^{1}(0, L)}+\left(w_{x t t}, \phi_{x}\right)-\left(w_{x x x}, \phi_{x}\right)+w_{t}(L, t) \phi(L)=-\left(\left[v_{x}+\frac{1}{2} w_{x}^{2}\right] w_{x}, \phi_{x}\right), \forall \phi \in V
\end{gathered}
$$

and the boundary conditions

$$
\left[v_{x}+\frac{1}{2} w_{x}^{2}\right](L, t)=-\varepsilon^{\alpha} v_{t}(L, t) \text { and } w_{x x}(L, t)=-w_{x t}(L, t), \text { a.e., } t>0 .
$$

Observe that strong solutions, as above, are not quite classical solutions of the system (4.1). Indeed, the first equation in (4.1), the boundary conditions in $x=0$ and the first two boundary conditions in $x=L$ are satisfied in the classical sense, but the second equation and the last boundary condition in $x=L$ are satisfied in a weaker, variational sense.

Let us analyze weak, finite energy solutions of (4.1): As in [11], we shall say that $t \mapsto U(t):[0, \infty) \rightarrow H$ is a weak solution of (4.1) if there is a sequence $U_{n}(t)$ of strong solutions such that $U_{n} \rightarrow U$ in $C([0, T] ; H)$ for each $T>0$. In what concerns weak solutions, taking into account that the set

$$
\vartheta=\left\{\begin{array}{l}
\left(v_{0}, v_{1}, w_{0}, w_{1}\right) \in\left[H^{2}(0, L) \cap V\right] \times V \times\left[H^{3}(0, L) \cap W\right] \times W: \\
v_{0, x}(L)+\frac{1}{2}\left(w_{0, x}(L)\right)^{2}=-\varepsilon^{\alpha} v_{1}(L) \text { and } w_{0, x x}(L)=-w_{1, x x}(L)
\end{array}\right\}
$$

is dense in $H$ in the strong topology, the following result was obtained:

Theorem 4.2 (Weak solutions). Let $\left(v_{0}, v_{1}, w_{0}, w_{1}\right) \in H$. Then, system (4.1) has a unique weak solution with

$$
\left\{v, v_{t}\right\} \in C\left([0, \infty) ; V \times L^{2}(0, L)\right),\left\{w, w_{t}\right\} \in C([0, \infty) ; W \times V) .
$$

Moreover, the time derivative of $E_{\varepsilon}(t)$ associated to solutions of (4.1) satisfies

$$
\frac{\mathrm{d} E_{\varepsilon}}{\mathrm{d} t}(t)=-\varepsilon^{\alpha} v_{t}^{2}(L, t)-w_{t}^{2}(L, t)-w_{x t}^{2}(L, t) .
$$

As a consequence of the energy dissipation law above

$$
E_{\varepsilon}(t) \leq E_{\varepsilon}(0)
$$

for all $t \geq 0$. This implies that, for any $\varepsilon>0, \quad\left\|\left(v, v_{t}, w, w_{t}\right)\right\|_{H}$ is bounded for $t \geq 0$ since $E_{\varepsilon}(t)$ is equivalent to $\left\|\left(v, v_{t}, w, w_{t}\right)\right\|_{H}$ for all $\varepsilon>0$. Furthermore, it could be shown that weak solutions are in fact strong solutions if the initial data have additional regularity and satisfy some compatibility conditions.

\subsection{Asymptotic limit as $\varepsilon \rightarrow \mathbf{0}$}

According to Section 4.1, problem (4.1) has an unique finite-energy solution. Moreover, the energy is decreasing in time. This provides uniform bounds for the solutions $\left\{v^{\varepsilon}, w^{\varepsilon}\right\}$ which allow us to pass to the limit as $\varepsilon \rightarrow 0$ as in Section 3.2. The only difficulty once again is to identify the weak limit of the nonlinear term. We have that

$$
w_{x}^{\varepsilon}\left(v_{x}^{\varepsilon}+\frac{1}{2}\left(w_{x}^{\varepsilon}\right)^{2}\right) \rightarrow \eta w_{x} \quad \text { weakly in } \quad L^{2}((0, L) \times(0, T))
$$


as $\varepsilon \rightarrow 0$, where

$$
\eta=\rho+\frac{1}{2} w_{x}^{2}
$$

and $\eta=\eta(t)$. Multiplying the first equation in (4.1) by $a(x)=\frac{x}{L}$ and integrating in space we obtain

$$
\begin{aligned}
\varepsilon \frac{\mathrm{d}^{2}}{\mathrm{~d} t^{2}} \int_{0}^{L} v^{\varepsilon} a(x) \mathrm{d} x & =\int_{0}^{L}\left[v_{x}^{\varepsilon}+\frac{1}{2}\left(w_{x}^{\varepsilon}\right)^{2}\right]_{x} a(x) \mathrm{d} x \\
& =-\varepsilon^{\alpha} v_{t}^{\varepsilon}(L, t)-\frac{1}{L} \int_{0}^{L}\left[v_{x}^{\varepsilon}+\frac{1}{2}\left(w_{x}^{\varepsilon}\right)^{2}\right] \mathrm{d} x
\end{aligned}
$$

The left-hand side tends to zero in $D^{\prime}(0, T)$ as $\varepsilon \rightarrow 0$. On the other hand, the right-hand side converges to $-\eta(t)$ because the boundary term tends to zero as $\varepsilon \rightarrow 0$. Indeed, thanks to the energy dissipation we have

$$
\varepsilon^{\alpha} \int_{0}^{T}\left|v_{t}^{\varepsilon}(L, t)\right|^{2} \mathrm{~d} t \leq E_{\varepsilon}(0)
$$

for all $T>0$, and therefore $\varepsilon^{\alpha / 2} v_{t}^{\varepsilon}(L, t)$ is bounded in $L^{2}(0, T)$. Consequently, $\varepsilon^{\alpha} v_{t}^{\varepsilon}(L, t) \rightarrow 0$ in $D^{\prime}(0, T)$ as $\varepsilon \rightarrow 0$ since $\alpha>0$. Thus, $\eta=0$ and we deduce that the limit of $w^{\varepsilon}$ satisfies:

$$
w \in L^{\infty}\left([0,+\infty ; W) \cap W^{1, \infty}([0,+\infty) ; V)\right.
$$

and

$$
-\int_{0}^{T}\left(w_{t}, \phi_{t}\right) \mathrm{d} t-\int_{0}^{T}\left(w_{x t}, \phi_{x t}\right) \mathrm{d} t+\int_{0}^{T}\left(w_{x x}, \phi_{x x}\right) \mathrm{d} t-\int_{0}^{T} w(L, t) \phi_{t}(L, t) \mathrm{d} t-\int_{0}^{T} w_{x}(L, t) \phi_{x t}(L, t) \mathrm{d} t=0,
$$

for all $\phi \in C([0, T] ; W)$, such that $\phi(x, 0)=\phi(x, T)=\phi_{x}(x, 0)=\phi(x, T)=0$. Furthermore arguing as in Section 3.1 we have that

$$
w(x, 0)=w_{0}(x), \quad w_{t}(x, 0)=w_{1}(x), \quad 0<x<L .
$$

Thus, $w \in L^{\infty}\left([0,+\infty ; W) \cap W^{1, \infty}([0,+\infty) ; V)\right.$ is a weak solution of

$$
\begin{cases}w_{t t}-w_{x x t t}+w_{x x x x}=0 & 0<x<L, t>0 \\ w(0, t)=w_{x}(0, t)=0, & t>0 \\ w_{x x}(L, t)=-w_{x t}(L, t), & t>0 \\ {\left[w_{x x x}-w_{x t t}\right](L, t)=w_{t}(L, t),} & t>0 \\ w(x, 0)=w_{0}(x), w_{t}(x, 0)=w_{1}(x), & 0<x<L .\end{cases}
$$

System (4.3) is the generator of a semigroup of contractions in $W \times V$ that we denote by $S(\cdot)$. Then, when $\left\{w_{0}, w_{1}\right\} \in W \times V$, (4.3) has an unique global weak solution $S(t)\left\{w_{0}, w_{1}\right\} \in C([0, \infty) ; W) \cap C^{1}([0, \infty) ; V)$. Note that the solution of (4.3) we have obtained in the limit is not, in principle, continuous in time with values in $W$ and $C^{1}$ with values in $V$. Thus, in order to conclude that the weak solution obtained in the limit is the semigroup solution we need a further argument.

The semigroup $S$ may be extended to a semigroup of contractions $T(t)$ in $E=V \times L^{2}(0, L)$, where $\left.T(t)\right|_{W \times V}=S(t)$. It is easy to see that the solution we have obtained in the limit coincides with the one the 
semigroup $T$ provides. Indeed, using the equation in (4.3) and the regularity $L^{\infty}\left([0,+\infty ; W) \cap W^{1, \infty}([0,+\infty) ; V)\right.$ it is easy to see that $w \in C\left([0,+\infty ; V) \cap C^{1}\left([0,+\infty) ; L^{2}(0, L)\right)\right.$ and that $w$ satisfies the weak formulation of $(4.3)$ corresponding to the solutions given by the semigroup $T(\cdot)$. Thus $\left(w(t), w_{t}(t)\right)=T(t)\left(w_{0}, w_{1}\right)$. Since, $\left(w_{0}, w_{1}\right)$ belongs to $W \times V$ and $T$ is an extension of the original semigroup $S(\cdot)$ we deduce that $\left(w(t), w_{t}(t)\right)=S(t)\left(w_{0}, w_{1}\right)$ and, as a consequence of that, the fact that $w \in C([0, \infty) ; W) \cap C^{1}([0, \infty) ; V)$.

Note that the energy in (4.3) is given by

$$
E(t)=\frac{1}{2} \int_{0}^{L}\left[w_{t}^{2}+w_{x t}^{2}+w_{x x}^{2}\right] \mathrm{d} x
$$

with the energy dissipation law being

$$
\frac{\mathrm{d} E}{\mathrm{~d} t}(t)=-w_{t}^{2}(L, t)-w_{x t}^{2}(L, t)
$$

There is a large literature on this model concerning existence, uniqueness and asymptotic behavior (see [9] and the references therein). In particular, it is by now well known that the energy of solutions of (4.3) tends exponentially uniformly to zero as $t$ goes to infinity. Thus, it is natural to expect the energy of solutions of (4.1) to tend exponentially to zero, uniformly with respect to the parameter $\varepsilon$.

\subsection{Uniform stabilization as $\varepsilon \rightarrow \mathbf{0}$}

The following result holds:

Theorem 4.3. Let $\{v, w\}$ be the global (weak) solution of system (4.1) obtained in Theorem 4.2. Assume that $0 \leq \alpha \leq 1$. Then, there exist positive constants $c, \mu>0$ such that

$$
E_{\varepsilon}(t) \leq c E(0) \mathrm{e}^{-\frac{\mu}{1+\varepsilon^{\alpha} E(0)} t}, \quad \text { a.e. } \quad t>0
$$

for all $0<\varepsilon<1$.

We will prove (4.4) for strong solutions since, by density, the result then holds for all weak solutions.

Remark. As a consequence of (4.4), by letting $\varepsilon \rightarrow 0$, we recover the exponential decay property of the solutions of (4.3). This is in agreement with the results obtained in [9].

Proof of Theorem 4.3. As before, in order to simplify notations we will write $w=w^{\varepsilon}, \quad v=v^{\varepsilon}$. Let $\delta>0$ and consider the "perturbed" energy $G_{\varepsilon, \delta}(t)$ given by

$$
G_{\varepsilon, \delta}(t)=E_{\varepsilon}(t)+\delta F_{\varepsilon}(t)
$$

and show that $G_{\varepsilon, \delta}$ enjoy the properties

$$
c_{1} G_{\varepsilon, \delta}(t) \leq E_{\varepsilon}(t) \leq c_{2} G_{\varepsilon, \delta}(t)
$$

and

$$
\frac{\mathrm{d} G_{\varepsilon, \delta}(t)}{\mathrm{d} t} \leq-c_{3} E_{\varepsilon}(t)
$$

for some positive constants $c_{1}, c_{2}$ and $c_{3}$ that will be computed explicitly below.

In this case $F_{\varepsilon}(t)$ is given by

$$
F_{\varepsilon}(t)=\int_{0}^{L}\left[x\left(\varepsilon v_{x} v_{t}+w_{x} w_{t}\right)+w_{x t}\left(x w_{x}\right)_{x}-\frac{5}{8}\left(w w_{t}+w_{x} w_{x t}\right)-\frac{\varepsilon}{4} v v_{t}\right] \mathrm{d} x .
$$


674

G.P. MENZALA ET AL.

The first step to prove (4.5) and (4.6) is to estimate the time derivative of $F_{\varepsilon}(t)$ :

$$
\begin{aligned}
\frac{\mathrm{d} F_{\varepsilon}}{\mathrm{d} t}(t)= & \int_{0}^{L} x\left[\varepsilon v_{x t} v_{t}+\varepsilon v_{x} v_{t t}+w_{x t} w_{t}+w_{x} w_{t t}\right] \mathrm{d} x \\
& +\int_{0}^{L}\left[w_{x t t}\left(x w_{x}\right)_{x}+w_{x t}\left(x w_{x t}\right)_{x}\right] \mathrm{d} x-\frac{5}{8} \int_{0}^{L}\left[w_{t}^{2}+w w_{t t}\right] \mathrm{d} x \\
& -\frac{5}{8} \int_{0}^{L}\left[w_{x t}^{2}+w_{x} w_{x t t}\right] \mathrm{d} x-\frac{\varepsilon}{4} \int_{0}^{L}\left[v_{t}^{2}+v v_{t t}\right] \mathrm{d} x
\end{aligned}
$$

Integration by parts give us that

$$
\int_{0}^{L} w_{x t t}\left(x w_{x}\right)_{x} \mathrm{~d} x-\frac{5}{8} \int_{0}^{L} w_{x} w_{x t t} \mathrm{~d} x=w_{x t t}(L, t)\left[L w_{x}(L, t)-\frac{5}{8} w(L, t)\right]-\int_{0}^{L} w_{x x t t}\left(x w_{x}-\frac{5}{8} w\right) \mathrm{d} x
$$

Now, we use the equations of system (4.1) and replace $\varepsilon v_{t t}$ and $w_{t t}$ to obtain

$$
\begin{aligned}
\frac{\mathrm{d} F_{\varepsilon}}{\mathrm{d} t}(t)= & \int_{0}^{L}\left(v_{x}+\frac{1}{2} w_{x}^{2}\right)_{x}\left(x v_{x}-\frac{1}{4} v\right) \mathrm{d} x \\
& +\int_{0}^{L}\left[-w_{x x x x}+\left[w_{x}\left(v_{x}+\frac{1}{2} w_{x}^{2}\right)\right]_{x}\right]\left(x w_{x}-\frac{5}{8} w\right) \mathrm{d} x \\
& +\varepsilon \int_{0}^{L} x v_{x t} v_{t} \mathrm{~d} x+\int_{0}^{L} x w_{x t} w_{t} \mathrm{~d} x+\int_{0}^{L} w_{x t}\left(x w_{x t}\right)_{x} \mathrm{~d} x \\
& -\frac{5}{8} \int_{0}^{L}\left[w_{t}^{2}+w_{x t}^{2}\right] \mathrm{d} x-\frac{\varepsilon}{4} \int_{0}^{L} v_{t}^{2} \mathrm{~d} x \\
& +w_{x t t}(L, t)\left[L w_{x}(L, t)-\frac{5}{8} w(L, t)\right] .
\end{aligned}
$$

The following identities (4.9) up to (4.15) are devoted to estimate the integrals in (4.8):

$$
\begin{aligned}
\int_{0}^{L}\left(v_{x}+\frac{1}{2} w_{x}^{2}\right)_{x}\left(x v_{x}-\frac{1}{4} v\right) \mathrm{d} x= & -\varepsilon^{\alpha} v_{t}(L, t)\left[L v_{x}(L, t)-\frac{1}{4} v(L, t)\right] \\
& -\int_{0}^{L}\left(v_{x}+\frac{1}{2} w_{x}^{2}\right)\left(x v_{x x}+\frac{3}{4} v_{x}\right) \mathrm{d} x \\
\int_{0}^{L}\left[\left(v_{x}+\frac{1}{2} w_{x}^{2}\right) w_{x}\right]_{x}\left(x w_{x}-\frac{5}{8} w\right) \mathrm{d} x= & \left(v_{x}+\frac{1}{2} w_{x}^{2}\right)(L, t) w_{x}(L, t)\left[L w_{x}(L, t)-\frac{5}{8} w(L, t)\right] \\
& -\int_{0}^{L}\left(v_{x}+\frac{1}{2} w_{x}^{2}\right) w_{x}\left(x w_{x x}+\frac{3}{8} w_{x}\right) \mathrm{d} x .
\end{aligned}
$$


Adding (4.9) with (4.10) we obtain the identity

$$
\begin{aligned}
\int_{0}^{L} & \left(v_{x}+\frac{1}{2} w_{x}^{2}\right)_{x}\left(x v_{x}-\frac{1}{4} v\right) \mathrm{d} x+\int_{0}^{L}\left[\left(v_{x}+\frac{1}{2} w_{x}^{2}\right) w_{x}\right]_{x}\left(x w_{x}-\frac{5}{8} w\right) \mathrm{d} x \\
= & -\frac{3}{4} \int_{0}^{L}\left(v_{x}+\frac{1}{2} w_{x}^{2}\right)^{2} \mathrm{~d} x-\frac{1}{2} \int_{0}^{L} x\left[\left(v_{x}+\frac{1}{2} w_{x}^{2}\right)^{2}\right]_{x} \mathrm{~d} x \\
& -\varepsilon^{\alpha} v_{t}(L, t)\left[L v_{x}(L, t)-\frac{1}{4} v(L, t)\right]+\left(v_{x}+\frac{1}{2} w_{x}^{2}\right)(L, t) w_{x}(L, t)\left[L w_{x}(L, t)-\frac{5}{8} w(L, t)\right] \\
\leq & -\frac{1}{4} \int_{0}^{L}\left(v_{x}+\frac{1}{2} w_{x}^{2}\right)^{2} \mathrm{~d} x+\frac{L}{2} \varepsilon^{\alpha} v_{t}^{2}(L, t)-\varepsilon^{\alpha} v_{t}(L, t)\left[L v_{x}(L, t)-\frac{1}{4} v(L, t)\right] \\
& +\left(v_{x}+\frac{1}{2} w_{x}^{2}\right)(L, t) w_{x}(L, t)\left[L w_{x}(L, t)-\frac{5}{8} w(L, t)\right] .
\end{aligned}
$$

We also have

$$
\begin{aligned}
-\int_{0}^{L} w_{x x x x}\left(x w_{x}-\frac{5}{8} w\right) \mathrm{d} x= & -w_{x x x}(L, t)\left[L w_{x}-\frac{5}{8} w\right](L, t)+\int_{0}^{L} w_{x x x}\left(x w_{x x}+\frac{3}{8} w_{x}\right) \mathrm{d} x \\
= & -w_{x x x}(L, t)\left[L w_{x}-\frac{5}{8} w\right](L, t)+\frac{L}{2} w_{x x}^{2}(L, t)+\frac{3}{8} w_{x x}(L, t) w_{x}(L, t) \\
& -\frac{7}{8} \int_{0}^{L} w_{x x}^{2} \mathrm{~d} x
\end{aligned}
$$

To conclude this step we compute three more terms in (4.8): First,

$$
\begin{aligned}
\varepsilon \int_{0}^{L} x v_{x t} v_{t} \mathrm{~d} x & =\frac{\varepsilon}{2} \int_{0}^{L} x\left[v_{t}^{2}\right]_{x} \mathrm{~d} x=\frac{\varepsilon}{2} L v_{t}^{2}(L, t)-\frac{\varepsilon}{2} \int_{0}^{L} v_{t}^{2} \mathrm{~d} x \\
& \leq \frac{\varepsilon^{\alpha}}{2} L v_{t}^{2}(L, t)-\frac{\varepsilon}{2} \int_{0}^{L} v_{t}^{2} \mathrm{~d} x
\end{aligned}
$$

since $0<\varepsilon<1$ and $0 \leq \alpha \leq 1$. On the other hand,

$$
\int_{0}^{L} x w_{x t} w_{t} \mathrm{~d} x=\frac{L}{2} w_{t}^{2}(L, t)-\frac{1}{2} \int_{0}^{L} w_{t}^{2} \mathrm{~d} x
$$

and

$$
\int_{0}^{L} w_{x t}\left(x w_{x t}\right)_{x} \mathrm{~d} x=\frac{L}{2} w_{x t}^{2}(L, t)+\frac{1}{2} \int_{0}^{L} w_{x t}^{2} \mathrm{~d} x .
$$


Returning to (4.8) and using equalities (4.11) up to (4.15), we obtain

$$
\begin{aligned}
\frac{\mathrm{d} F_{\varepsilon}}{\mathrm{d} t}(t) \leq & -\frac{1}{4} \int_{0}^{L}\left(v_{x}+\frac{1}{2} w_{x}^{2}\right)^{2} \mathrm{~d} x-\frac{7}{8} \int_{0}^{L} w_{x x}^{2} \mathrm{~d} x-\frac{3 \varepsilon}{4} \int_{0}^{L} v_{t}^{2} \mathrm{~d} x \\
& -\frac{1}{2} \int_{0}^{L} w_{t}^{2} \mathrm{~d} x+\frac{1}{2} \int_{0}^{L} w_{x t}^{2} \mathrm{~d} x-\frac{5}{8} \int_{0}^{L} w_{t}^{2} \mathrm{~d} x-\frac{5}{8} \int_{0}^{L} w_{x t}^{2} \mathrm{~d} x \\
& -\left(w_{x x x}-w_{x t t}-\left(v_{x}+\frac{1}{2} w_{x}^{2}\right) w_{x}\right)(L, t)\left(L w_{x}-\frac{5}{8} w\right)(L, t) \\
& -\varepsilon^{\alpha} v_{t}(L, t)\left[L v_{x}(L, t)-\frac{1}{4} v(L, t)\right]+\frac{L}{2} w_{x x}^{2}(L, t) \\
& +\frac{3}{8} w_{x x}(L, t) w_{x}(L, t)+\frac{L \varepsilon^{\alpha}}{2} v_{t}^{2}(L, t) \\
& +\frac{L}{2} w_{t}^{2}(L, t)+\frac{L}{2} w_{x t}^{2}(L, t) .
\end{aligned}
$$

In view of the boundary conditions in (4.1), the boundary terms can be estimated as follows:

$$
\begin{aligned}
\left|-\left(w_{x x x}-w_{x t t}-\left(v_{x}+\frac{1}{2} w_{x}^{2}\right) w_{x}\right)(L, t)\left(L w_{x}-\frac{5}{8} w\right)(L, t)\right| & =\left|-w_{t}(L, t)\right|\left|\left(L w_{x}-\frac{5}{8} w\right)(L, t)\right| \\
& \leq \frac{1}{\eta}\left|w_{t}(L, t)\right|^{2}+\eta c \int_{0}^{L} w_{x x}^{2} \mathrm{~d} x,
\end{aligned}
$$

for all $\eta>0$ where $c$ is a positive constant. Moreover,

$$
\left|\frac{3}{8} w_{x x}(L, t) w_{x}(L, t)\right|=\frac{3}{8}\left|-w_{x t}(L, t)\right|\left|w_{x}(L, t)\right| \leq \frac{c}{\eta}\left|w_{x t}(L, t)\right|^{2}+c \eta \int_{0}^{L} w_{x x}^{2} \mathrm{~d} x
$$

for all $\eta>0$. Finally, we have:

$$
\begin{aligned}
\varepsilon^{\alpha} v_{t}(L, t)\left[L v_{x}(L, t)-\frac{1}{4} v(L, t)\right] & \leq \varepsilon^{\alpha} L\left|v_{t}(L, t) v_{x}(L, t)\right|+\frac{\varepsilon^{\alpha}}{4}\left|v_{t}(L, t) v(L, t)\right| \\
& \leq \varepsilon^{\alpha} c\left[\frac{2}{\eta} v_{t}^{2}(L, t)+\eta v_{x}^{2}(L, t)+\eta \int_{0}^{L} v_{x}^{2} \mathrm{~d} x\right]
\end{aligned}
$$

for some positive constant $c$.

To bound the term $v_{x}^{2}(L, t)$ we proceed as follows:

$$
\left|v_{x}(L, t) w_{x}^{2}(L, t)\right| \leq \frac{1}{2} v_{x}^{2}(L, t)+\frac{1}{2} w_{x}^{4}(L, t)
$$

consequently

$$
\left(v_{x}(L, t)+\frac{1}{2} w_{x}^{2}(L, t)\right)^{2} \geq \frac{1}{2} v_{x}^{2}(L, t)-\frac{1}{4} w_{x}^{4}(L, t)
$$


This inequality, combined with the boundary conditions at $x=L$ in (4.1), gives us that

$$
\begin{aligned}
v_{x}^{2}(L, t) & \leq 2\left(v_{x}(L, t)+\frac{1}{2} w_{x}^{2}(L, t)\right)^{2}+\frac{1}{4} w_{x}^{4}(L, t) \\
& \left.\leq 2 \varepsilon^{2 \alpha} v_{t}^{2}(L, t)+c E_{\varepsilon}(0)\right) \int_{0}^{L} w_{x x}^{2} \mathrm{~d} x \\
& \leq 2 \varepsilon^{\alpha} v_{t}^{2}(L, t)+c E_{\varepsilon}(0) \int_{0}^{L} w_{x x}^{2} \mathrm{~d} x
\end{aligned}
$$

because $0<\varepsilon<1$ and $\alpha>0$. Now, to bound the term $\int_{0}^{L} v_{x}^{2} \mathrm{~d} x$ in (4.19) we can proceed as before, to obtain

$$
\begin{aligned}
\int_{0}^{L} v_{x}^{2} \mathrm{~d} x & \leq 2 \int_{0}^{L}\left[\left(v_{x}+\frac{1}{2} w_{x}^{2}\right)^{2}+\frac{1}{4} w_{x}^{4}\right] \mathrm{d} x \\
& \leq 2 \int_{0}^{L}\left(v_{x}+\frac{1}{2} w_{x}^{2}\right)^{2} \mathrm{~d} x+c\left(\int_{0}^{L} w_{x x}^{2} \mathrm{~d} x\right)^{2} \\
& \leq 2 \int_{0}^{L}\left(v_{x}+\frac{1}{2} w_{x}^{2}\right)^{2} \mathrm{~d} x+c E_{\varepsilon}(0) \int_{0}^{L} w_{x x}^{2} \mathrm{~d} x
\end{aligned}
$$

where $c$ is a positive constant. Returning to (4.19), from (4.20) and (4.21) it follows that

$\varepsilon^{\alpha} v_{t}(L, t)\left[L v_{x}(L, t)-\frac{1}{4} v(L, t)\right] \leq 2 c \varepsilon^{\alpha}\left[\left(\frac{1}{\eta}+\eta \varepsilon^{\alpha}\right) v_{t}^{2}(L, t)+\eta \int_{0}^{L}\left(v_{x}+\frac{1}{2} w_{x}^{2}\right)^{2} \mathrm{~d} x+\eta E_{\varepsilon}(0) \int_{0}^{L} w_{x x}^{2} \mathrm{~d} x\right]$.

Thus, returning to (4.16) and combining all the estimates above (4.17-4.22) we have

$$
\begin{aligned}
\frac{\mathrm{d} F_{\varepsilon}(t)}{\mathrm{d} t} \leq & -\frac{3 \varepsilon}{4} \int_{0}^{L} v_{t}^{2} \mathrm{~d} x-\frac{9}{8} \int_{0}^{L} w_{t}^{2} \mathrm{~d} x-\frac{1}{8} \int_{0}^{L} w_{x t}^{2} \mathrm{~d} x \\
& -\left[\frac{7}{8}-\eta c\left(1+\varepsilon^{\alpha} E_{\varepsilon}(0)\right)\right] \int_{0}^{L} w_{x x}^{2} \mathrm{~d} x \\
& -\left[\frac{1}{4}-\varepsilon^{\alpha} c \eta\right] \int_{0}^{L}\left(v_{x}+\frac{1}{2} w_{x}^{2}\right)^{2} \mathrm{~d} x+\left[\frac{L}{2}+\frac{1}{\eta}\right] w_{t}^{2}(L, t) \\
& +\left[L+\frac{c}{\eta}\right] w_{x t}^{2}(L, t)+\left[\frac{1}{\eta}+\eta \varepsilon^{\alpha}\right] c \varepsilon^{\alpha} v_{t}^{2}(L, t) .
\end{aligned}
$$

We may now estimate the time-derivative of $G_{\varepsilon, \delta}$ defined in (4.5). First we recall that

$$
\frac{\mathrm{d} E_{\varepsilon}}{\mathrm{d} t}(t)=-\varepsilon^{\alpha} v_{t}^{2}(L, t)-w_{x t}^{2}(L, t)-w_{t}^{2}(L, t) .
$$


Then, using (4.23) we obtain

$$
\begin{aligned}
\frac{\mathrm{d} G_{\varepsilon, \delta}(t)}{\mathrm{d} t} \leq & -\frac{3 \varepsilon \delta}{4} \int_{0}^{L} v_{t}^{2} \mathrm{~d} x-\frac{9 \delta}{8} \int_{0}^{L} w_{t}^{2} \mathrm{~d} x-\frac{\delta}{8} \int_{0}^{L} w_{x t}^{2} \mathrm{~d} x \\
& -\delta\left[\frac{7}{8}-\eta c\left(1+\varepsilon^{\alpha} E_{\varepsilon}(0)\right)\right] \int_{0}^{L} w_{x x}^{2} \mathrm{~d} x \\
& -\delta\left[\frac{1}{4}-\varepsilon^{\alpha} c \eta\right] \int_{0}^{L}\left(v_{x}+\frac{1}{2} w_{x}^{2}\right)^{2} \mathrm{~d} x-\left[1-\delta c\left(\frac{L}{2}+\frac{1}{\eta}\right)\right] w_{t}^{2}(L, t) \\
& -\left[1-\delta\left(L+\frac{c}{\eta}\right)\right] w_{x t}^{2}(L, t)-\left[\varepsilon^{\alpha}-\varepsilon^{\alpha} \delta c\left(\frac{1}{\eta}+\eta \varepsilon^{\alpha}\right)\right] v_{t}^{2}(L, t)
\end{aligned}
$$

for some positive constant $c$. Now, we choose $\eta$ of the order of

$$
\eta=\frac{\lambda}{1+\varepsilon^{\alpha} E(0)}
$$

with $\lambda>0$ (small enough but independent of $\varepsilon$ and $E_{\varepsilon}(0)$ ) so that (4.24) reads as follows:

$$
\begin{aligned}
\frac{\mathrm{d} G_{\varepsilon, \delta}(t)}{\mathrm{d} t} \leq & -\frac{3 \varepsilon \delta}{4} \int_{0}^{L} v_{t}^{2} \mathrm{~d} x+\frac{9 \delta}{8} \int_{0}^{L} w_{t}^{2} \mathrm{~d} x-\frac{\delta}{8} \int_{0}^{L} w_{x t}^{2} \mathrm{~d} x-\frac{\delta}{2} \int_{0}^{L} w_{x x}^{2} \mathrm{~d} x \\
& -\frac{\delta}{2} \int_{0}^{L}\left(v_{x}+\frac{1}{2} w_{x}^{2}\right)^{2} \mathrm{~d} x-\left[1-\delta c\left(\frac{L}{2}+\frac{1}{\eta}\right)\right] w_{t}^{2}(L, t) \\
& -\left[1-\delta\left(L+\frac{c}{\eta}\right)\right] w_{x t}^{2}(L, t)-\left[\varepsilon^{\alpha}-\varepsilon^{\alpha} \delta c\left(\frac{1}{\eta}+\eta \varepsilon^{\alpha}\right)\right] v_{t}^{2}(L, t) .
\end{aligned}
$$

We also choose $\delta>0$ such that

$$
\begin{aligned}
1-\delta c\left(\frac{L}{2}+\frac{\left(1+\varepsilon^{\alpha} E_{\varepsilon}(0)\right)}{\lambda}\right) \geq 0, \quad 1-\delta\left[L+\frac{c\left(1+\varepsilon^{\alpha} E_{\varepsilon}(0)\right)}{\lambda}\right] & \geq 0 \\
& \text { and } \varepsilon^{\alpha}-\varepsilon^{\alpha} \delta c\left(\frac{1+\varepsilon^{\alpha} E_{\varepsilon}(0)}{\lambda}+\frac{\varepsilon^{\alpha} \lambda}{1+\varepsilon^{\alpha} E_{\varepsilon}(0)}\right) \geq 0 .
\end{aligned}
$$

If these conditions hold, in view of (4.26), we deduce that

$$
\frac{\mathrm{d} G_{\varepsilon, \delta}}{\mathrm{d} t}(t) \leq-\frac{\delta}{4} E_{\varepsilon}(t) .
$$

Observe that (4.27) holds if

$$
\delta \leq \frac{c}{1+\varepsilon^{\alpha} E_{\varepsilon}(0)}
$$

with $c>0$ independent of $0<\varepsilon<1$ and the solution. Moreover, proceeding as in (3.37) we have

$$
\left|F_{\varepsilon}(t)\right| \leq c\left[E_{\varepsilon}(t)+\varepsilon E_{\varepsilon}(t)^{2}\right] \leq c\left[1+\varepsilon E_{\varepsilon}(0)\right] E_{\varepsilon}(t)
$$

where $c$ is independent of $0<\varepsilon<1$. Consequently,

$$
\left|G_{\varepsilon, \delta}(t)-E_{\varepsilon}(t)\right|=\delta\left|F_{\varepsilon}(t)\right| \leq c \delta\left[1+\varepsilon E_{\varepsilon}(0)\right] E_{\varepsilon}(t),
$$


and, for $c$ sufficiently small,

$$
\left|G_{\varepsilon, \delta}(t)-E_{\varepsilon}(t)\right| \leq \frac{1}{2} E_{\varepsilon}(t)
$$

Thus, taking (4.28) and (4.30) into account we obtain

$$
E_{\varepsilon}(t) \leq \tilde{c} E_{\varepsilon}(0) \mathrm{e}^{-\frac{\mu}{1+\varepsilon^{\alpha} E_{\varepsilon}(0)} t}
$$

and $\mu$ and $\tilde{c}$ are positive constants independent of $0<\varepsilon<1$ and the solution.

\section{Plate models: internal DAMPing}

Similar problems arise for vibrating plates in two space dimensions. In this section, we consider the full dynamic von Kármán system for a $2-D$ plate with internal damping. Let $\Omega$ be a bounded domain of $\mathbb{R}^{2}$ with smooth boundary. Let us consider the deflections of a $2-D$ plate occupying the domain $\Omega$. We denote by $U=(u, v)$ the in-plane displacement of the plate where both $u$ and $v$ depend on the space variables $(x, y) \in \Omega$ and time $t>0$. We represent by $w=w(x, y, t)$ the vertical displacement of the plate. The full von Kármán dynamical model for large deflections of the plate, with internal damping and perturbed by a parameter $\varepsilon>0$, reads as follows (see [8] and the references therein):

$$
\begin{gathered}
\varepsilon U_{t t}=\operatorname{Div}\left(\mathcal{C}^{\varepsilon}[e(U)+f(\nabla w)]\right)-\varepsilon^{\alpha} U_{t} \text { in } \Omega \times(0, \infty), \\
w_{t t}+\Delta^{2} w-\Delta w_{t t}=\operatorname{div}\left(\mathcal{C}^{\varepsilon}[e(U)+f(\nabla w)] \nabla w\right)-w_{t}+\Delta w_{t} \text { in } \Omega \times(0, \infty) .
\end{gathered}
$$

In $(5.1), \mathcal{C}^{\varepsilon}$ denotes the fourth order tensor defined by

$$
\mathcal{C}^{\varepsilon}[e]=\frac{E}{d\left(1-\mu^{2}\right)}[\mu(\operatorname{Tr} e) I+\varepsilon(1-\mu) e]
$$

for any $e$ in $S$, the space of $2 \times 2$ symmetric matrices, where $I$ is the identity matrix and (Tr $e$ ) denotes the trace of $e$. Moreover, $d>0$ is the density of the plate, $E>0$ denotes the Young modulus and $0<\mu<1$ the Poisson's ratio. In (5.1), Div denotes the vector valued divergence of a matrix and div stands for the scalar divergence of a vector field.

On the other hand, $e[U]=\frac{1}{2}\left(\nabla U+(\nabla U)^{T}\right)$ and the nonlinearity $f: \mathbb{R}^{2} \rightarrow S$ in $(5.1,5.2)$ is defined as $f(\xi)=\frac{1}{2} \xi \otimes \xi$ for all $\xi \in \mathbb{R}^{2}$. We complement system $(5.1,5.2)$ with Dirichlet boundary conditions

$$
U=0, \quad w=\frac{\partial w}{\partial \eta}=0 \quad \text { on } \quad \partial \Omega \times(0, \infty)
$$

and initial conditons

$$
\begin{cases}U(x, y, 0)=(u(x, y, 0), v(x, y, 0))=\left(u_{0}(x, y), v_{0}(x, y)\right) & \text { in } \Omega \\ U_{t}(x, y, 0)=\left(u_{t}(x, y, 0), v_{t}(x, y, 0)\right)=\left(u_{1}(x, y), v_{1}(x, y)\right) & \text { in } \Omega \\ w(x, y, 0)=w_{0}(x, y), w_{t}(x, y, 0)=w_{1}(x, y) & \text { in } \Omega .\end{cases}
$$

We want to analyse the solution $\left\{U=U^{\varepsilon}, w=w^{\varepsilon}\right\}$ of $(5.1-5.5)$ as $\varepsilon \rightarrow 0$ and $t \rightarrow+\infty$.

In (5.4) $\partial / \partial \eta$ denotes the normal derivative, $\eta$ being the unit outward normal to $\Omega$. 
The total energy associated with the above system is given by

$$
\begin{aligned}
E_{\varepsilon}(t)= & \frac{1}{2} \int_{\Omega}\left\{\varepsilon\left(u_{t}^{\varepsilon}\right)^{2}+\varepsilon\left(v_{t}^{\varepsilon}\right)^{2}+\left(w_{t}^{\varepsilon}\right)^{2}+\left|\nabla w_{t}^{\varepsilon}\right|^{2}+\left|\Delta w^{\varepsilon}\right|^{2}\right\} \mathrm{d} A \\
& +\frac{1}{2}\left(\mathcal{C}^{\varepsilon}\left(e\left[U^{\varepsilon}\right]+f\left(\nabla w^{\varepsilon}\right)\right), e\left[U^{\varepsilon}\right]+f\left(\nabla w^{\varepsilon}\right)\right),
\end{aligned}
$$

where $U^{\varepsilon}=\left(u^{\varepsilon}, v^{\varepsilon}\right)$. The last term on the right hand side of (5.6) has to be interpreted as the inner product in $\left(L^{2}(\Omega)\right)^{4}$. Direct calculations using the definitions given above show that

$$
e\left[U^{\varepsilon}\right]+f\left(\nabla w^{\varepsilon}\right)=\left[b_{i j}^{\varepsilon}\right],
$$

where

$$
b_{11}^{\varepsilon}=u_{x}^{\varepsilon}+\frac{1}{2}\left|w_{x}^{\varepsilon}\right|^{2} ; \quad b_{22}^{\varepsilon}=v_{y}^{\varepsilon}+\frac{1}{2}\left|w_{y}^{\varepsilon}\right|^{2} ; \quad b_{12}^{\varepsilon}=b_{21}^{\varepsilon}=\frac{1}{2}\left(u_{y}^{\varepsilon}+v_{x}^{\varepsilon}+w_{x}^{\varepsilon} w_{y}^{\varepsilon}\right)
$$

and

$$
\mathcal{C}^{\varepsilon}\left(\left[b_{i_{j}}\right]\right)=\gamma\left\{\mu\left[\begin{array}{cc}
b_{11}^{\varepsilon}+b_{22}^{\varepsilon} & 0 \\
0 & b_{11}^{\varepsilon}+b_{22}^{\varepsilon}
\end{array}\right]+\varepsilon(1-\mu)\left[\begin{array}{ll}
b_{11}^{\varepsilon} & b_{12}^{\varepsilon} \\
b_{21}^{\varepsilon} & b_{22}^{\varepsilon}
\end{array}\right]\right\}
$$

Consequently

$$
\begin{aligned}
\left(\mathcal{C}^{\varepsilon}\left(\left[b_{i_{j}}^{\varepsilon}\right]\right),\left[b_{i j}^{\varepsilon}\right]\right)_{\left(L^{2}(\Omega)\right)^{4}} & \gamma \mu\left\|u_{x}^{\varepsilon}+v_{y}^{\varepsilon}+\frac{1}{2}\left|\nabla w^{\varepsilon}\right|^{2}\right\|_{L^{2}(\Omega)}^{2} \\
& +\varepsilon \gamma(1-\mu)\left[\left\|b_{11}^{\varepsilon}\right\|_{L^{2}(\Omega)}^{2}+\left\|b_{22}^{\varepsilon}\right\|_{L^{2}(\Omega)}^{2}+\frac{1}{2}\left\|v_{x}^{\varepsilon}+u_{y}^{\varepsilon}+w_{x}^{\varepsilon} w_{y}^{\varepsilon}\right\|_{L^{2}(\Omega)}^{2}\right]>0
\end{aligned}
$$

since $\gamma>0$ and $0<\mu<1$, which shows that $\mathcal{C}^{\varepsilon}$ is positive definite.

We deduce from the above discussion that, when we are dealing with smooth solutions, the total energy $E_{\varepsilon}(t)$ given by (5.6) is positive definite and decreasing according to the law

$$
\frac{\mathrm{d} E_{\varepsilon}}{\mathrm{d} t}(t)=-\varepsilon^{\alpha}\left\|U_{t}^{\varepsilon}\right\|_{\left(L^{2}(\Omega)\right)^{2}}^{2}-\left\|w_{t}^{\varepsilon}\right\|_{L^{2}(\Omega)}^{2}-\left\|\nabla w_{t}^{\varepsilon}\right\|_{L^{2}(\Omega)}^{2} .
$$

Consequently

$$
E_{\varepsilon}(t) \leq E_{\varepsilon}(0), \quad \text { a.e. } \quad t \geq 0 .
$$

The so-called damped Berger-Timoshenko's model is much simpler (see [1] and [4]). It consists on a single scalar equation for the vertical displacement $w$. Namely

$$
w_{t t}+\Delta^{2} w-\Delta w_{t t}-c\left(\int_{\Omega}|\nabla w|^{2} \mathrm{~d} A\right) \Delta w-w_{t}+\Delta w_{t}=0
$$

where $c$ is a positive constant.

The corresponding boundary and initial conditions are now

$$
w=\frac{\partial w}{\partial \eta}=0 \quad \text { on } \quad \partial \Omega \times(0, \infty)
$$


and

$$
w(x, y, 0)=w_{0}(x, y), \quad w_{t}(x, y, 0)=w_{1}(x, y) \quad \text { in } \quad \Omega .
$$

The energy associated to (5.9-5.11) is given by

$$
E(t)=\frac{1}{2} \int_{\Omega}\left(w_{t}^{2}+\left|\nabla w_{t}\right|^{2}+|\Delta w|^{2}\right) \mathrm{d} A+\frac{c}{4}\left(\int_{\Omega}|\nabla w|^{2} \mathrm{~d} A\right)^{2}
$$

and the energy dissipation law is then

$$
\frac{\mathrm{d} E}{\mathrm{~d} t}(t)=-\int_{\Omega} w_{t}^{2} \mathrm{~d} A-\int_{\Omega}\left|\nabla w_{t}\right|^{2} \mathrm{~d} A
$$

Existence and uniqueness of solutions for (5.9-5.11) is by now well known and can be obtained by classical methods. It turns out that for any $\left(w_{0}, w_{1}\right)$ belonging to $H_{0}^{2}(\Omega) \times H_{0}^{1}(\Omega)$ system (5.9-5.11) has a unique global solution $w$ in the class $C\left([0, \infty) ; H_{0}^{2}(\Omega)\right) \cap C^{1}\left([0, \infty) ; H_{0}^{1}(\Omega)\right)$. Existence of finite energy solutions of the full von Kármán system (5.1-5.5) is also well known. In [10] (see also [20]) it was proved that for the conservative case, finite energy solutions are unique. In the context our damped system given $\left(U_{0}, U_{1}\right) \in\left(H_{0}^{1}(\Omega)\right)^{2} \times\left(L^{2}(\Omega)\right)^{2}$ and $\left(w_{0}, w_{1}\right) \in H_{0}^{2}(\Omega) \times H_{0}^{1}(\Omega)$ system $(5.1,5.2,5.4,5.5)$ admits a unique finite energy solution in the class

$$
\left\{\begin{array}{l}
U^{\varepsilon} \in L^{\infty}\left(0, \infty ;\left(H_{0}^{2}(\Omega)\right)^{2}\right) \cap W^{1, \infty}\left(0, \infty ;\left(L^{2}(\Omega)\right)^{2}\right) . \\
w^{\varepsilon} \in L^{\infty}\left(0, \infty ; H_{0}^{2}(\Omega)\right) \cap W^{1, \infty}\left(0, \infty ; H_{0}^{1}(\Omega)\right) .
\end{array}\right.
$$

The same proof of uniqueness applies in the present case. Solutions of both the full von Kármán system and Berger-Timoshenko's equation being unique there is no ambiguity in analyzing the proximity of solutions of both models as $\varepsilon \rightarrow 0$. In the engineering literature, there is a formal procedure named Berger's approximation where such proximity is claimed (see, for instance [13], Sect. 7, 6.1). The mathematical justification of this limit in the conservative case was proved in [18]. The same argument allows to pass to the limit in the present dissipative case.

For Berger-Timoshenko and von Kármán's models considered here, the energy decays exponentially as $t \rightarrow$ $+\infty$. Our goal here is to show that, actually, the decay rate is uniform (as $\varepsilon \rightarrow 0$ ), provided $0<\alpha \leq 1$ and locally uniform for bounded data when $\alpha=0$. Note however that when $\alpha=0$, once again, the limit is of different nature.

\subsection{Uniform stabillization as $\varepsilon \rightarrow \mathbf{0}$}

The following result holds

Theorem 5.1. Let $\{U, w\}$ be the global solution of (5.1-5.5) as in (5.12). Assume that $0 \leq \alpha \leq 1$. Then, there exist positive constans $c>0, \quad \mu>0$, such that

$$
E_{\varepsilon}(t) \leq c E_{\varepsilon}(0) \mathrm{e}^{-\frac{\mu}{1+\varepsilon^{\alpha} E_{\varepsilon}(0)}} t, \quad \text { a.e. } \quad t>0
$$

for all $0<\varepsilon<1$.

We will prove (5.13) for strong solutions since, by density, and the lower semicontinuity of the energy functional, the result then holds for all weak solutions. 
Proof. We will prove the result in three steps:

Step 1. Let us consider the functional

$$
F_{\varepsilon}(t)=\varepsilon \int_{\Omega} U^{\varepsilon} \cdot U_{t}^{\varepsilon} \mathrm{d} A+\frac{1}{2} \int_{\Omega}\left[w^{\varepsilon} w_{t}^{\varepsilon}+\nabla w^{\varepsilon} \cdot \nabla w_{t}^{\varepsilon}\right] \mathrm{d} A .
$$

Using the equations $(5.1,5.2)$ and taking into account that we are dealing with regular solutions we obtain that

$$
\begin{aligned}
\frac{\mathrm{d} F_{\varepsilon}}{\mathrm{d} t}(t)= & \varepsilon \int_{\Omega}\left|U_{t}^{\varepsilon}\right|^{2} \mathrm{~d} A+\varepsilon \int_{\Omega} U^{\varepsilon} \cdot U_{t t}^{\varepsilon} \mathrm{d} A+\frac{1}{2} \int_{\Omega}\left[\left(w_{t}^{\varepsilon}\right)^{2}+\left|\nabla w_{t}^{\varepsilon}\right|^{2}\right] \mathrm{d} A \\
& +\frac{1}{2} \int_{\Omega}\left[w^{\varepsilon} w_{t t}^{\varepsilon}+\nabla w^{\varepsilon} \cdot \nabla w_{t t}^{\varepsilon}\right] \mathrm{d} A \\
= & \varepsilon \int_{\Omega}\left|U_{t}^{\varepsilon}\right|^{2} \mathrm{~d} A+\int_{\Omega} U^{\varepsilon} \cdot\left[\operatorname{Div}\left(C^{\varepsilon}\left[e\left(U^{\varepsilon}\right)+f\left(\nabla w^{\varepsilon}\right)\right]\right)\right] \mathrm{d} A \\
& -\varepsilon^{\alpha} \int_{\Omega} U^{\varepsilon} \cdot U_{t}^{\varepsilon} \mathrm{d} A+\frac{1}{2} \int_{\Omega}\left[\left(w_{t}^{\varepsilon}\right)^{2}+\left|\nabla w_{t}^{\varepsilon}\right|^{2}\right] \mathrm{d} A \\
& +\frac{1}{2} \int_{\Omega} w^{\varepsilon}\left[-\Delta^{2} w^{\varepsilon}+\operatorname{div}\left(C^{\varepsilon}\left(e\left(U^{\varepsilon}\right)+f\left(\nabla w^{\varepsilon}\right)\right) \nabla w^{\varepsilon}\right)-w_{t}^{\varepsilon}+\Delta w_{t}^{\varepsilon}\right] \mathrm{d} A .
\end{aligned}
$$

Integration by parts and using the explicit expression of $C^{\varepsilon}\left[e\left(U^{\varepsilon}\right)+f\left(\nabla w^{\varepsilon}\right)\right]$ implies that

$$
\begin{aligned}
\int_{\Omega} U^{\varepsilon} \cdot \operatorname{Div}\left[C^{\varepsilon}\left(e\left(U^{\varepsilon}\right)+f\left(\nabla w^{\varepsilon}\right)\right)\right] \mathrm{d} A+\frac{1}{2} \int_{\Omega} w^{\varepsilon} \operatorname{div}\left(\left(C^{\varepsilon}\left(e\left(U^{\varepsilon}\right)+f\left(\nabla w^{\varepsilon}\right)\right) \nabla w^{\varepsilon}\right) \mathrm{d} A=\right. \\
-\left(C^{\varepsilon}\left(e\left(U^{\varepsilon}\right)+f\left(\nabla w^{\varepsilon}\right)\right), e\left(U^{\varepsilon}\right)+f\left(\nabla w^{\varepsilon}\right)\right)_{\left(L^{2}(\Omega)\right)^{4} .}
\end{aligned}
$$

Observe that the right hand side of (5.15) was explcitly calculated in (5.7). In order to get an estimate for the right hand side of (5.14) we use bounds for $\int_{\Omega} w^{\varepsilon} w_{t}^{\varepsilon} \mathrm{d} A, \quad \int_{\Omega} w^{\varepsilon} \Delta w_{t}^{\varepsilon} \mathrm{d} A$ and $\int_{\Omega} U^{\varepsilon} \cdot U_{t}^{\varepsilon} \mathrm{d} A$ as follows: For any $\eta>0$ we have that

$$
\begin{aligned}
\left|\int_{\Omega} w^{\varepsilon} w_{t}^{\varepsilon} \mathrm{d} A\right| & \leq c \int_{\Omega}\left[\eta\left|\Delta w^{\varepsilon}\right|^{2} \mathrm{~d} A+\frac{1}{\eta}\left(w_{t}^{\varepsilon}\right)^{2}\right] \mathrm{d} A \\
\left|\int_{\Omega} w^{\varepsilon} \Delta w_{t}^{\varepsilon} \mathrm{d} A\right| & \leq c \int_{\Omega}\left[\eta\left|\Delta w^{\varepsilon}\right|^{2}+\frac{1}{\eta}\left|\nabla w_{t}^{\varepsilon}\right|^{2}\right] \mathrm{d} A
\end{aligned}
$$

and

$$
\varepsilon^{\alpha}\left|\int_{\Omega} U^{\varepsilon} \cdot U_{t}^{\varepsilon} \mathrm{d} A\right| \leq \frac{\varepsilon^{\alpha}}{2 \eta} \int_{\Omega}\left[\left(u_{t}^{\varepsilon}\right)^{2}+\left(v_{t}^{\varepsilon}\right)^{2}\right] \mathrm{d} A+\frac{\varepsilon^{\alpha} \eta}{2} \int_{\Omega}\left[\left(u^{\varepsilon}\right)^{2}+\left(v^{\varepsilon}\right)^{2}\right] \mathrm{d} A
$$

for some positive constant $c$. Next, we use Korn's inequality (see [6]) to obtain

$$
\begin{aligned}
\int_{\Omega}\left[\left(u^{\varepsilon}\right)^{2}+\left(v^{\varepsilon}\right)^{2}\right] \mathrm{d} A & \leq c_{1} \int_{\Omega}\left[\left(u_{x}^{\varepsilon}\right)^{2}+\left(v_{y}^{\varepsilon}\right)^{2}+\left(v_{x}^{\varepsilon}+u_{y}^{\varepsilon}\right)^{2}\right] \mathrm{d} A \\
& \leq c_{2}\left\{\left\|b_{11}^{\varepsilon}\right\|_{L^{2}(\Omega)}^{2}+\left\|b_{22}^{\varepsilon}\right\|_{L^{2}(\Omega)}^{2}+\left\|b_{12}^{\varepsilon}\right\|_{L^{2}(\Omega)}^{2}+\int_{\Omega}\left|\nabla w^{\varepsilon}\right|^{2} \mathrm{~d} A\right\} \\
& \leq c_{2}\left\{\left\|b_{11}^{\varepsilon}\right\|_{L^{2}(\Omega)}^{2}+\left\|b_{22}^{\varepsilon}\right\|_{L^{2}(\Omega)}^{2}+\left\|b_{12}^{\varepsilon}\right\|_{L^{2}(\Omega)}^{2}+E_{\varepsilon}(0) \int_{\Omega}\left|\Delta w^{\varepsilon}\right|^{2} \mathrm{~d} A\right\}
\end{aligned}
$$

for some positive constant $c_{2}$. 
Combining (5.15-5.19) with (5.14) we obtain the estimate

$$
\begin{aligned}
\frac{\mathrm{d} F_{\varepsilon}}{\mathrm{d} t} \leq & \left(1+\frac{\varepsilon^{\alpha-1}}{2 \eta}\right) \varepsilon \int_{\Omega}\left[\left(u_{t}^{\varepsilon}\right)^{2}+\left(v_{t}^{\varepsilon}\right)^{2}\right] \mathrm{d} A \\
& +\left(\frac{1}{2}+\frac{c}{\eta}\right) \int_{\Omega}\left[\left(w_{t}^{\varepsilon}\right)^{2}+\left|\nabla w_{t}^{\varepsilon}\right|^{2}\right] \mathrm{d} A \\
& -\frac{1}{2}\left(1-c \eta\left[4+\varepsilon^{\alpha} E_{\varepsilon}(0)\right]\right) \int_{\Omega}\left|\Delta w^{\varepsilon}\right|^{2} \mathrm{~d} A \\
& -\left(\gamma(1-\mu)-\frac{\varepsilon^{\alpha-1} \eta c}{2}\right) \varepsilon\left[\left\|b_{11}^{\varepsilon}\right\|_{L^{2}}^{2}+\left\|b_{22}^{\varepsilon}\right\|_{L^{2}}^{2}+\left\|b_{12}^{\varepsilon}\right\|_{L^{2}}^{2}\right] \\
& -\gamma \mu \int_{\Omega}\left(u_{x}^{\varepsilon}+v_{y}^{\varepsilon}+\frac{1}{2}|\nabla w|^{2}\right)^{2} \mathrm{~d} A .
\end{aligned}
$$

Step 2. Now, we set

$$
G_{\varepsilon, \delta}(t)=E_{\varepsilon}(t)+\delta F_{\varepsilon}(t)
$$

where $\delta>0, \quad E_{\varepsilon}(t)$ is as in (5.6) and $F_{\varepsilon}(t)$ as above. Using (5.8) and (5.20) we deduce that

$$
\begin{aligned}
\frac{\mathrm{d} G_{\varepsilon, \delta}(t)}{\mathrm{d} t} \leq & -\left[\varepsilon^{\alpha-1}-\delta-\frac{\delta \varepsilon^{\alpha-1}}{2 \eta}\right] \varepsilon \int_{\Omega}\left[\left(u_{t}^{\varepsilon}\right)^{2}+\left(v_{t}^{\varepsilon}\right)^{2}\right] \mathrm{d} A \\
& -\frac{\delta}{2}\left[1-c \eta\left(4+\varepsilon^{\alpha} E_{\varepsilon}(0)\right)\right] \int_{\Omega}\left|\Delta w^{\varepsilon}\right|^{2} \mathrm{~d} A \\
& -\left[1-\delta\left(\frac{1}{2}+\frac{c}{\eta}\right)\right] \int_{\Omega}\left[\left(w_{t}^{\varepsilon}\right)^{2}+\left|\nabla w_{t}^{\varepsilon}\right|^{2}\right] \mathrm{d} A \\
& -\delta \gamma \mu \int_{\Omega}\left(u_{x}^{\varepsilon}+v_{y}^{\varepsilon}+\frac{1}{2}\left|\nabla w^{\varepsilon}\right|^{2}\right)^{2} \mathrm{~d} A \\
& -\delta \varepsilon\left[\gamma(1-\mu)-\frac{\varepsilon^{\alpha-1}}{2} \eta c\right]\left\{\left\|b_{11}^{\varepsilon}\right\|_{L^{2}}^{2}+\left\|b_{22}^{\varepsilon}\right\|_{L^{2}}^{2}+\left\|b_{12}^{\varepsilon}\right\|_{L^{2}}^{2}\right\}
\end{aligned}
$$

Now we choose $\eta$ of the order

$$
\eta=\frac{\lambda}{1+\varepsilon^{\alpha} E_{\varepsilon}(0)}
$$

with $\lambda>0$ sufficiently small but independent of $\varepsilon$ and $E_{\varepsilon}(0)$. From (5.21) then follows

$$
\begin{aligned}
\frac{\mathrm{d} G_{\varepsilon, \delta}}{\mathrm{d} t}(t) \leq & -\left[\varepsilon^{\alpha-1}-\delta-\frac{\delta}{2 \lambda} \varepsilon^{\alpha-1}\left(1+\varepsilon^{\alpha} E_{\varepsilon}(0)\right)\right] \int_{\Omega}\left[\left(u_{t}^{\varepsilon}\right)^{2}+\left(v_{t}^{\varepsilon}\right)^{2}\right] \mathrm{d} A \\
& -\frac{\delta}{4} \int_{\Omega}\left|\Delta w^{\varepsilon}\right|^{2} \mathrm{~d} A-\left[1-\delta\left(\frac{1}{2}+\frac{c}{\lambda}\left(1+\varepsilon^{\alpha} E_{\varepsilon}(0)\right)\right)\right] \int_{\Omega}\left[\left(w_{t}^{\varepsilon}\right)^{2}+\left|\nabla w_{t}^{\varepsilon}\right|^{2}\right] \mathrm{d} A \\
& -\frac{\delta}{2}\left(C^{\varepsilon}\left(e\left(U^{\varepsilon}\right)+f\left(\nabla w^{\varepsilon}\right)\right), e\left(U^{\varepsilon}\right)+f\left(\nabla w^{\varepsilon}\right)\right)_{\left[L^{2}(\Omega)\right]^{4}}
\end{aligned}
$$


We now impose the following restrictions on $\delta$ :

$$
\begin{gathered}
\delta\left(\frac{1}{2}+\frac{c}{\lambda}\left(1+\varepsilon^{\alpha} E_{\varepsilon}(0)\right)\right)<1 \\
\varepsilon^{\alpha-1}-\delta-\frac{\delta}{2 \lambda} \varepsilon^{\alpha-1}\left(1+\varepsilon^{\alpha} E_{\varepsilon}(0)\right) \geq \frac{1}{2} .
\end{gathered}
$$

If these conditions hold, in view of (5.22), we deduce that

$$
\frac{\mathrm{d} G_{\varepsilon, \delta}(t)}{\mathrm{d} t} \leq-\min \{1, \delta\} E_{\varepsilon}(t) .
$$

Conditions (5.23) and (5.24) hold if we take $\delta>0$ such that

$$
\delta \leq c \min \left\{\left(1+\varepsilon^{\alpha} E_{\varepsilon}(0)\right)^{-1}, \varepsilon^{\alpha-1}\right\}
$$

with $c>0$ small enough but independent of $0<\varepsilon<1$ and $E_{\varepsilon}(0)$.

Step 3. To conclude the proof we need to compare $E_{\varepsilon}$ and $G_{\varepsilon, \delta}$.

Using $(5.16,5.17)$ and $(5.19)$ we deduce that

$$
\begin{aligned}
\left|F_{\varepsilon}(t)\right| & \leq \frac{\varepsilon}{2} \int_{\Omega}\left[\left(u_{t}^{\varepsilon}\right)^{2}+\left(v_{t}^{\varepsilon}\right)^{2}\right] \mathrm{d} A+\frac{\varepsilon}{2} \int_{\Omega}\left[\left(u^{\varepsilon}\right)^{2}+\left(v^{\varepsilon}\right)^{2}\right] \mathrm{d} A+c \int_{\Omega}\left[\left|\nabla w_{t}^{\varepsilon}\right|^{2}+\left|\Delta w^{\varepsilon}\right|^{2}\right] \mathrm{d} A \\
& \leq C\left[E_{\varepsilon}(t)+\varepsilon E_{\varepsilon}(0) E_{\varepsilon}(t)\right]=C\left[1+\varepsilon E_{\varepsilon}(0)\right] E_{\varepsilon}(t)
\end{aligned}
$$

with $c>0$ independent of $\varepsilon(0<\varepsilon<1)$ and the solution. Consequently,

$$
\left|G_{\varepsilon, \delta}(t)-E_{\varepsilon}(t)\right|=\delta\left|F_{\varepsilon}(t)\right| \leq c \delta\left(1+\varepsilon E_{\varepsilon}(0)\right) E_{\varepsilon}(t) .
$$

Choosing $\delta>0$ as above and $c>0$ smaller (if needed) we can guarantee that

$$
\left|G_{\varepsilon, \delta}(t)-E_{\varepsilon}(t)\right| \leq \frac{1}{2} E_{\varepsilon}(t) .
$$

Thus, in view of (5.25), it follows that

$$
\frac{\mathrm{d} G_{\varepsilon, \delta}}{\mathrm{d} t}(t) \leq-\frac{1}{2} \min (1, \delta) G_{\varepsilon, \delta}(t) .
$$

As a consequence of (5.28) and (5.29) we deduce the existence of positive constants $\tilde{c}>0$ and $\tilde{\mu}>0$ such that

$$
E_{\varepsilon}(t) \leq \tilde{c} E_{\varepsilon}(0) \mathrm{e}^{-\frac{\tilde{\tilde{\mu}}}{1+\varepsilon^{\alpha} E(0)} t}, \quad \text { a.e. } t>0
$$

for every weak solution of $(5.1,5.2,5.4,5.5)$ and every $0<\varepsilon<1$.

\section{Further COMments AND OPEN PROBlems}

The methods developed in this paper allow us to address other situations. In this section we briefly discuss some of them. We analyse first the case where $\alpha=0$ for beams with internal and boundary damping, and for plates with internal damping. We finally mention some open problems on the subject. 


\subsection{Asymptotic limit as $\varepsilon \rightarrow 0$ when $\alpha=0$ : Internal damping}

For any $\varepsilon>0$ we consider

$$
\left\{\begin{array}{l}
\varepsilon v_{t t}=\left[v_{x}+\frac{1}{2} w_{x}^{2}\right]_{x}-v_{t} \\
w_{t t}+w_{x x x x}-w_{x x t t}=\left[\left(v_{x}+\frac{1}{2} w_{x}^{2}\right) w_{x}\right]_{x}-w_{t}+w_{x x t}
\end{array}\right.
$$

in $0<x<L, \quad t>0$ with boundary and initial conditions

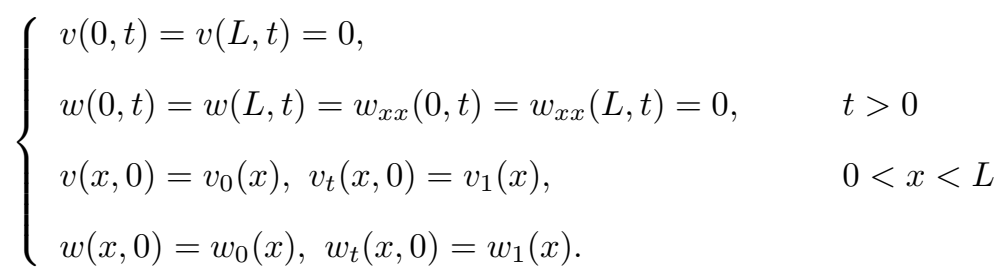

This corresponds to system (3.1) with $\alpha=0$.

The energy $E_{\varepsilon}(t)$ is given by

$$
E_{\varepsilon}(t)=\frac{1}{2} \int_{0}^{L}\left\{\varepsilon v_{t}^{2}+w_{t}^{2}+w_{x t}^{2}+w_{x x}^{2}+\left(v_{x}+\frac{1}{2} w_{x}^{2}\right)^{2}\right\} \mathrm{d} x
$$

and satisfies the law

$$
\frac{\mathrm{d} E_{\varepsilon}(t)}{\mathrm{d} t}=-\int_{0}^{L}\left(v_{t}^{2}+w_{t}^{2}+w_{x t}^{2}\right) \mathrm{d} x \leq 0 .
$$

Let $H$ be as in (3.2). We analyse the asymptotic limit of this system as $\varepsilon$ tends to zero.

Due to $(6.3)$, we know that the following sequences (in $\varepsilon$ ) remain bounded in $L^{\infty}\left(0,+\infty ; L^{2}(0, L)\right)$ :

$$
\left\{\sqrt{\varepsilon} v_{t}^{\varepsilon}\right\}, \quad\left\{v_{x}^{\varepsilon}+\frac{1}{2}\left(w_{x}^{\varepsilon}\right)^{2}\right\}, \quad\left\{w_{t}^{\varepsilon}\right\}, \quad\left\{w_{x t}^{\varepsilon}\right\}, \quad\left\{w_{x x}^{\varepsilon}\right\} .
$$

We proceed as in Section 3.1: We extract subsequences as in (3.6-3.8). We denote by $z$ (resp. w) the weak limit of $v^{\varepsilon}\left(\right.$ resp. $\left.w^{\varepsilon}\right)$.

We use Aubin-Lions' compactness criteria (see [21]) to deduce that

$$
w^{\varepsilon} \rightarrow w \quad \text { strongly in } \quad L^{\infty}\left(0, T ; H^{2-\delta}(0, L)\right)
$$

as $\varepsilon \rightarrow 0$, for any $\delta>0$ and $T<+\infty$. Thus, we obtain that

$$
w_{x}^{\varepsilon}\left(v_{x}^{\varepsilon}+\frac{1}{2}\left(w_{x}^{\varepsilon}\right)^{2}\right) \rightarrow w_{x} \eta \quad \text { weakly in } \quad L^{2}((0, L) \times(0, T))
$$

as $\varepsilon \rightarrow 0$, where $\eta$ is the weak limit in $L^{2}((0, L) \times(0, T))$ of $v_{x}^{\varepsilon}+\frac{1}{2}\left(w_{x}^{\varepsilon}\right)^{2}$.

We have

$$
\eta=v_{x}+\frac{1}{2}\left(w_{x}\right)^{2}
$$


We also have

$$
\varepsilon v_{t t}^{\varepsilon} \rightarrow 0 \quad \text { weakly in } \quad H^{-1}\left(0, T ; L^{2}(0, L)\right)
$$

as $\varepsilon \rightarrow 0$.

Thus, from the first equation in $(6.1,6.5)$ and $(6.6)$ it follows that

$$
z_{t}=\left[z_{x}+\frac{1}{2} w_{x}^{2}\right]_{x}
$$

Thus, the limit

$$
(z, w) \in L^{\infty}\left(0, \infty ; H_{0}^{1}(0, L)\right) \times\left[L^{\infty}\left(0, \infty ; H^{2} \cap H_{0}^{1}(0, L)\right) \cap W^{1, \infty}\left(0, \infty ; L^{2}(0, L)\right)\right]
$$

is a weak solution of

$$
\left\{\begin{array}{l}
z_{t}=\left[z_{x}+\frac{1}{2} w_{x}^{2}\right]_{x} \\
w_{t t}+w_{x x x x}-w_{x x t t}=\left[\left(z_{x}+\frac{1}{2} w_{x}^{2}\right) w_{x}\right]_{x}-w_{t}+w_{x x t}
\end{array}\right.
$$

in $0<x<L, \quad t>0$ with boundary and initial conditions

$$
\begin{cases}z(0, t)=z(L, t)=0 & \\ w(0, t)=w(L, t)=w_{x x}(0, t)=w_{x x}(L, t)=0 & \\ z(x, 0)=v_{0}(x), & 0<x<L \\ w(x, 0)=w_{0}(x), w_{t}(x, 0)=w_{1}(x), & 0<x<L .\end{cases}
$$

Note that, in the limit process, we keep the initial data $v_{0}$ for $z$ but not for its velocity $z_{t}$.

System $(6.4,6.5)$ is the coupling between a parabolic equation and a fourth order hyperbolic equation. In this sense it is similar to a system of thermoelasticity.

The total energy associated with problem $(6.4,6.5)$ is given by

$$
E(t)=\frac{1}{2} \int_{0}^{L}\left\{w_{t}^{2}+w_{x t}^{2}+w_{x x}^{2}+\left(z_{x}+\frac{1}{2} w_{x}^{2}\right)^{2}\right\} \mathrm{d} x
$$

and satisfies

$$
\frac{\mathrm{d} E(t)}{\mathrm{d} t}=-\int_{0}^{L}\left\{z_{t}^{2}+w_{t}^{2}+w_{x t}^{2}\right\} \mathrm{d} x
$$

Problem $(6.4,6.5)$ is well-posed in the space

$$
Y=\left[H^{2} \cap H_{0}^{1}(0, L)\right] \times H_{0}^{1}(0, L) \times H_{0}^{1}(0, L)
$$

whenever the initial data $\left(w_{0}, w_{1}, v_{0}\right) \in Y$.

According to Theorem 3.1 and passing to the limit as $\varepsilon \rightarrow 0$, the following exponential decay rate is obtained for the solutions of $(6.4,6.5)$ :

$$
E(t) \leq c E(0) \mathrm{e}^{-\frac{\mu}{1+E(0)} t}
$$

for all $t>0$ where $c$ and $\mu$ are positive constants. 


\subsection{Asymptotic limit as $\varepsilon \rightarrow 0$ when $\alpha=0$ : Boundary damping}

Let us now consider problem (4.1) with $\alpha=0$.

Following the discussion in Section 4.2, to pass to the limit as $\varepsilon$ tends to zero, we have to identify the weak limit of the nonlinear term $\left\{w_{x}^{\varepsilon}\left(v_{x}^{\varepsilon}+\frac{1}{2}\left(w_{x}^{\varepsilon}\right)^{2}\right)\right\}$. We know that

$$
w_{x}^{\varepsilon}\left(v_{x}^{\varepsilon}+\frac{1}{2}\left(w_{x}^{\varepsilon}\right)^{2}\right) \rightarrow \eta w_{x} \quad \text { weakly in } \quad L^{2}((0, L) \times(0, T))
$$

as $\varepsilon \rightarrow 0$ and

$$
\eta=v_{x}+\frac{1}{2} w_{x}^{2}
$$

where $w^{\varepsilon} \rightarrow w$ strongly in $L^{\infty}\left(0, T ; H^{2-\delta}(0, L)\right)$ and $v^{\varepsilon} \rightarrow v$ weakly in $L^{2}\left(0, T ; H^{1}(0, L)\right)$ as $\varepsilon \rightarrow 0$.

Let $a(x)=\frac{x}{L}, \quad 0 \leq x \leq L$. Multiply the first equation in (4.1) by $a(x)$ and integrate in space to obtain, after integration by parts and using the boundary conditions,

$$
\begin{aligned}
\varepsilon \frac{\mathrm{d}^{2}}{\mathrm{~d} t^{2}} \int_{0}^{L} v^{\varepsilon} a(x) \mathrm{d} x & =\int_{0}^{L}\left(v_{x}^{\varepsilon}+\frac{1}{2}\left(w_{x}^{\varepsilon}\right)^{2}\right)_{x} a(x) \mathrm{d} x \\
& =-v_{t}^{\varepsilon}(L, t)-\frac{1}{L} \int_{0}^{L}\left(v_{x}^{\varepsilon}+\frac{1}{2}\left(w_{x}^{\varepsilon}\right)^{2}\right) \mathrm{d} x \\
& =-v_{t}^{\varepsilon}(L, t)-\frac{1}{L} v^{\varepsilon}(L, t)-\frac{1}{2 L} \int_{0}^{L}\left(w_{x}^{\varepsilon}\right)^{2} \mathrm{~d} x .
\end{aligned}
$$

Due to the energy dissipation (see Th. 4.2) we know that $\left\{v_{t}^{\varepsilon}(L, t)\right\}$ is bounded in $L^{2}(0,+\infty)$. Therefore we can extract a subsequence of $v^{\varepsilon}(L, t)$ which converges weakly in $H_{l o c}^{1}(0, \infty)$ to some function $\xi=\xi(t)$ as $\varepsilon \rightarrow 0$. Then $\xi$ satisfies the ODE

$$
\xi_{t}+\frac{1}{L}\left[\xi+\frac{1}{2 L} \int_{0}^{L} w_{x}^{2} \mathrm{~d} x\right]=0
$$

and

$$
L \eta(t)=\xi(t)+\frac{1}{2} \int_{0}^{L} w_{x}^{2} \mathrm{~d} x
$$

The left hand side of (6.6) tends to zero weakly in $H^{-1}\left(0, T ; L^{2}(0, L)\right)$ as $\varepsilon \rightarrow 0$.

Moreover, since $\left\{v^{\varepsilon}(L, t)\right\}$ is bounded in $H^{1}(0, T)$, it follows that $v^{\varepsilon}(L, t) \rightarrow \xi(t)$ in $C([0, T])$ for any finite $T>0$. Consequently $\xi(0)=v_{0}(L)$. Thus, the limit system in case $\alpha=0$ is this case

$$
\left\{\begin{array}{l}
\xi^{\prime}+\frac{1}{L}\left[\xi+\frac{1}{2} \int_{0}^{L} w_{x}^{2} \mathrm{~d} x\right]=0, \quad t>0 \\
w_{t t}+w_{x x x x}-w_{x x t t}-\frac{1}{L}\left[\xi(t)+\frac{1}{2} \int_{0}^{L} w_{x}^{2} \mathrm{~d} x\right] w_{x x}=0 \quad \text { in }(0, L) \times(0,+\infty)
\end{array}\right.
$$


with boundary conditions

$$
\left\{\begin{array}{l}
w(0, t)=w_{x}(0, t)=0 \\
w_{x x}(L, t)=-w_{x t}(L, t) \\
{\left[w_{x x x}-w_{x t t}-\frac{1}{L}\left(\xi(t)+\frac{1}{2} \int_{0}^{L} w_{x}^{2} \mathrm{~d} x\right) w_{x}\right](L, t)=w_{t}(L, t)}
\end{array}\right.
$$

and initial conditions

$$
\xi(0)=v_{0}(L), \quad w(x, 0)=w_{0}(x), \quad w_{t}(x, 0)=w_{1}(x), \quad 0<x<L .
$$

The energy of the limit system is given by

$$
E(t)=\frac{1}{2} \int_{0}^{L}\left(w_{t}^{2}+w_{x t}^{2}+w_{x x}^{2}\right) \mathrm{d} x+\frac{1}{2 L}\left[\xi^{2}+\frac{1}{2} \int_{0}^{L} w_{x}^{2} \mathrm{~d} x\right]^{2}
$$

which is the natural limit of the energies of the $\varepsilon$-system.

A direct calculation shows that

$$
\frac{\mathrm{d} E(t)}{\mathrm{d} t}=-w_{t}^{2}(L, t)-w_{x t}^{2}(L, t)-\left|\xi^{\prime}\right|^{2} .
$$

Passing to the limit as $\varepsilon$ tends to zero on the uniform exponential decay estimates obtained in Section 4 for $\alpha=0$ we deduce that the energy $E$ of the limit system satisfies

$$
E(t) \leq c E(0) \mathrm{e}^{-\frac{\mu}{1+E(0)} t}
$$

for all $t>0$ where $c$ and $\mu$ are positive constants independent of the solution.

\subsection{Dirichlet boundary condition on $v$ and boundary damping: An open problem}

Let us consider again the $1-D$ beam model with different dissipative boundary conditions. More precisely, let us consider the system

$$
\begin{cases}\varepsilon v_{t t}=\left[v_{x}+\frac{1}{2} w_{x}^{2}\right]_{x}, & 0<x<L, t>0 \\ w_{t t}+w_{x x x x}-w_{x x t t}=\left[\left(v_{x}+\frac{1}{2} w_{x}^{2}\right) w_{x}\right]_{x}, & 0<x<L, t>0 \\ v(0, t)=v(L, t)=0 & t>0 \\ w(0, t)=w_{x}(L, t)=0, & \\ w_{x x}(L, t)=-w_{x t}(L, t) & \\ {\left[w_{x x x}-w_{x t t}-\left(v_{x}+\frac{1}{2} w_{x}^{2}\right) w_{x}\right](L, t)=w_{t}(L, t)} & 0<x<L . \\ v(x, 0)=v_{0}(x), v_{t}(x, 0)=v_{1}(x) & \\ & \end{cases}
$$


Let us consider the total energy $E_{\varepsilon}(t)$ as in (3.4). We can verify that

$$
\frac{\mathrm{d} E_{\varepsilon}(t)}{\mathrm{d} t}=-w_{t}^{2}(L, t)-w_{x t}^{2}(L, t)
$$

for all $t \geq 0$, therefore $E_{\varepsilon}(t)$ is decreasing and (6.11) provides uniform (as $\varepsilon \rightarrow 0$ ) bounds on the solutions $\left\{v=v^{\varepsilon}, w=w^{\varepsilon}\right\}$ which allow as to pass to the limit as $\varepsilon \rightarrow 0$.

Again the main difficulty is to identify the limit of the term: $\eta=\rho+\frac{1}{2} w_{x}^{2}$. If we proceed as in the previous sections, we deduce that $\eta_{x}=0$ therefore $\eta=\eta(t)$. Integration in space implies that

$$
L \eta(t)=\int_{0}^{L} \rho \mathrm{d} x+\frac{1}{2} \int_{0}^{L} w_{x}^{2} \mathrm{~d} x=\frac{1}{2} \int_{0}^{L} w_{x}^{2} \mathrm{~d} x
$$

since

$$
\int_{0}^{L} \rho \mathrm{d} x=\lim _{\varepsilon \rightarrow 0} \int_{0}^{L} v_{x}^{\varepsilon} \mathrm{d} x=v^{\varepsilon}(L, t)-v^{\varepsilon}(0, t)=0 .
$$

Thus, the (weak) limit system as $\varepsilon \rightarrow 0$ of problem (6.10) (in $w$ ) is as follows

$$
\left\{\begin{array}{l}
w_{t t}+w_{x x x x}-w_{x x t t}-\frac{1}{2 L}\left(\int_{0}^{L} w_{x}^{2} \mathrm{~d} x\right) w_{x x}=0 \\
w(0, t)=w_{x}(0, t)=0 \\
w_{x x}(L, t)=-w_{x t}(L, t) \\
{\left[w_{x x x}-w_{x t t}-\frac{1}{2}\left(\int_{0}^{L} w_{x}^{2} \mathrm{~d} x\right) w_{x}\right](L, t)=w_{t}(L, t)} \\
w(x, 0)=w_{0}(x), w_{t}(x, 0)=w_{1}(x)
\end{array}\right.
$$

for $0<x<L$ and $t>0$.

It is known (see [14]) that, for $\varepsilon>0$ fixed, the total energy of the solution $w=w(x, t)$ of model (6.12) decays exponentially as $t \rightarrow+\infty$ on bounded sets of initial data. But the analysis of the asymptotic behavior of the full system (6.10) and searching for uniform decay rates as $\varepsilon \rightarrow 0$ presents new difficulties. In view of the structure of system (6.10) and in order to obtain an estimate similar to (4.24) would be natural to use the multiplier $x u_{x}-\beta u$ where $\beta$ is a suitable positive constant. However, when we do this some extra terms appear with respect to previous calculations. Therefore, the uniform (with respect to $\varepsilon$ ) exponential decay of solutions of system (6.10) is an open problem.

\subsection{Asymptotic limit as $\varepsilon \rightarrow 0$ : Plate model with internal damping}

Let us consider the plate model we studied in Section 5 in case $\alpha=0$. We can prove that the solution $\left\{U^{\varepsilon}, w^{\varepsilon}\right\}$ of problem $(5.1,5.2,5.4,5.5)$ in case $\alpha=0$ converges (weakly) to the solution $\{U, w\}$ of the following 
system

$$
\begin{cases}U_{t}=\operatorname{Div}\left(C^{0}[e(U)+f(\nabla w)]\right) & \text { in } \Omega \times(0,+\infty) \\ w_{t t}+\Delta^{2} w-\Delta w_{t t}=\operatorname{div}\left(C^{0}[e(U)+f(\nabla w)] \nabla w\right)-w_{t}+\Delta w_{t} & \text { in } \Omega \times(0,+\infty) \\ U \cdot \eta=0, \quad w=\frac{\partial w}{\partial \eta}=0 \quad \text { on } \quad \partial \Omega(0,+\infty) & \text { in } \Omega \\ U(x, y, 0)=(u(x, y, 0), v(x, y, 0))=\left(u_{0}(x, y), v_{0}(x,, y)\right) & \text { in } \Omega \\ w(x, y, 0)=w_{0}(x, y), w_{t}(x, y, 0)=w_{1}(x, y) & \end{cases}
$$

where $C^{0}(A)=\gamma \mu \operatorname{Tr}(A) I$ for any $2 \times 2$ symmetric matrix $A$ (see (5.3) with $\varepsilon=0$ ). We could also rewrite $C^{0}(e(U)+f(\nabla w))$ explicitly:

$$
C^{0}(e(U)+f(\nabla w))=\gamma \mu\left[\begin{array}{cc}
u_{x}+v_{y}+\frac{1}{2}|\nabla w|^{2} & 0 \\
0 & u_{x}+v_{y}+\frac{1}{2}|\nabla w|^{2}
\end{array}\right]
$$

The total energy associated to the above system is given by

$$
E_{\varepsilon}(t)=\frac{1}{2} \int_{\Omega}\left\{w_{t}^{2}+\left|\nabla w_{t}\right|^{2}+|\Delta w|^{2}\right\} \mathrm{d} A+\frac{\gamma \mu}{2} \int_{\Omega}\left(u_{x}+v_{y}+\frac{1}{2}|\nabla w|^{2}\right)^{2} \mathrm{~d} A
$$

and satisfies

$$
\frac{\mathrm{d} E_{\varepsilon}(t)}{\mathrm{d} t}=-\int_{\Omega}\left\{\left|U_{t}\right|^{2}+w_{t}^{2}+\left|\nabla w_{t}\right|^{2}\right\} \mathrm{d} A \leq 0
$$

Note that the energy $E$ does not provide a complete estimate for $\nabla U$. Indeed, we simply get control on div $U$. It is precisely for that reason that the boundary condition one gets on $U$ is $U \cdot \eta=0$. Moreover, the natural space for $U$ is then

$$
V=\left\{U \in\left[L^{2}(\Omega)\right]^{2}: \operatorname{div} U \in L^{2}(\Omega), \quad U \cdot \eta=0 \quad \text { on } \quad \partial \Omega\right\}
$$

If we take initial data $\left(w_{0}, w_{1}\right) \in\left[H^{2}(\Omega) \cap H_{0}^{1}(\Omega)\right] \times H_{0}^{1}(\Omega)$ and $U(x, y, 0)=\left(u_{0}, v_{0}\right) \in V$ then the above system is globally well-posed in the space

$$
Y=\left[H^{2}(\Omega) \cap H_{0}^{1}(\Omega)\right] \times H_{0}^{1}(\Omega) \times V .
$$

According to Theorem 5.1, the following decay rate is obtained for weak solutions

$$
E(t) \leq c E(0) \mathrm{e}^{-\frac{\tilde{\mu}}{1+E(0)} t}, \quad \text { a.e. } \quad t>0
$$

where $c$ and $\tilde{\mu}$ are positive constants.

\subsection{Plate models with boundary damping}

The problems considered in this paper may also be addressed for full von Kármán systems with various boundary conditions including feedback terms which are useful for stabilization purposes (see [16] and the references therein). In particular, it would be natural to analyse the convergence of the solution of this system 
to a Berger-Timoshenko's system with boundary damping as the parameter $\varepsilon$ tends to zero. The question of the uniform decay would also be worth considering. This is an open problem.

Acknowledgements. G. Perla Menzala was partially supported by a grant of CNPq (Brasil) and PRONEX at LNCC/MCT (Brasil). Part of this work was done while this author was visiting the Departamento de Matemática Aplicada of the Universidad Complutense de Madrid. He express his thanks for their kind hospitality and support. The second author was supported by a Post-Doctoral grant BEX1432/99-0 of the CAPES (MCT-Brasil) and by FUJB (Fundação Universitária José Bonifácio) (Brasil) during his visit to Universidad Complutense de Madrid where this work was concluded. The third author was supported by FAPERJ (Brasil) during a visit to the LNCC/MCT and by grant PB96-0663 of the DGES (Spain) and the TMR project of the EU "Homogenization and Multiple Scales".

\section{REFERENCES}

[1] J.M. Ball, Initial-boundary value problems for an extensible beam. J. Math. Anal. Appl. 41 (1973) 69-90.

[2] Ph. Ciarlet, Mathematical elasticity, Vol. II. Theory of plates. Stud. Math. Appl. 27 (1997).

[3] A. Cimetière, G. Geymonat, H. Le Dret, A. Raoult and Z. Tutek, Asymptotic theory and analysis for displacements and stress distribution in nonlinear elastic straight slender rods. J. Elasticity 19 (1988) 111-161.

[4] R.W. Dickey, Free vibrations and dynamic buckling of the extensible beam. J. Math. Anal. Appl. 29 (1970) $443-454$.

[5] A. Haraux and E. Zuazua, Decay estimates for some damped hyperbolic equations. Arch. Rational Mech. Anal. 100 (1998) $191-206$.

[6] V.A. Kondratiev and O.A. Oleinik, Hardy's and Korn's type inequalities and their applications. Rendiconti di Matematica VII (1990) 641-666.

[7] V. Komornik and E. Zuazua, A direct method for the boundary stabilization of the wave equation. J. Math. Pures Appl. (9) 69 (1990) 33-55.

[8] J.E. Lagnese, Boundary stabilization of thin plates. SIAM Stud. Appl. Math., Philadelphia (1989).

[9] J.E. Lagnese, Recent progress in exact boundary controllability and uniform stability of thin beams and plates. Lect. Notes in Pure and Appl. Math. 128, Dekker, New York (1991) 61-111.

[10] I. Lasiecka, Weak, classical and intermediate solutions to full von Kármán system of dynamic nonlinear elasticity. Appl. Anal. 68 (1998) 121-145.

[11] J.E. Lagnese and G. Leugering, Uniform stabilization of a nonlinear beam by nonlinear boundary feedback. J. Differential Equations 91 (1991) 355-388.

[12] J.L. Lions, Perturbations singulières dans les problèmes aux limites et contrôle optimal. Springer-Verlag, Berlin, in Lectures Notes in Math. 323 (1973).

[13] A.H. Nayfeh and D.T. Mook, Nonlinear oscillations. Wiley-Interscience, New York (1989).

[14] A.F. Pazoto and G.P. Menzala, Uniform stabilization of a nonlinear beam model with thermal effects and nonlinear boundary dissipation. Funkcial. Ekvac. 43 (2000) 339-360.

[15] J.P. Puel and M. Tucsnak, Boundary stabilization for the von Karman equations. SIAM J. Control Optim. 33 (1995) 255-273

[16] J.P. Puel and M. Tucsnak, Global existence of the full von Kármán system. Appl. Math. Optim. 34 (1996) $139-160$.

[17] G.P. Menzala and E. Zuazua, The beam equation as a limit of $1-D$ nonlinear von Kármán model. Appl. Math. Lett. 12 (1999) 47-52.

[18] G.P. Menzala and E. Zuazua, Timoshenko's beam equation as limit of a nonlinear one-dimensional von Kármán system. Proc. Roy. Soc. Edinburg Sect. A 130 (2000) 855-875.

[19] G.P. Menzala and E. Zuazua, Timoshenko's plate equation as a singular limit of the dynamical von Kármán system. J. Math. Pures Appl. (9) 79 (2000) 73-94.

[20] V.I. Sedenko, On the uniqueness theorem for generalized solutions of initial-boundary problems for the Marguerre-Vlasov vibrations of shallow shells with clamped boundary conditions. Appl. Math. Optim. 39 (1999) 309-326.

[21] J. Simon, Compact sets in the space $L^{p}(0, T ; B)$. Ann. Mat. Pura Appl. (4) CXLVI (1987) 65-96.

[22] L. Trabucho de Campos and J. Viaño, Mathematical modelling of rods. Handbook of numerical analysis, Vol. IV, North Holland, Amsterdam (1996) 487-974.

[23] E. Zuazua, Stability and decay for a class of nonlinear hyperbolic problems. Asymptot. Anal. 1 (1988) 1-28. 